\title{
DENOMINATORS OF CLUSTER VARIABLES
}

\author{
ASLAK BAKKE BUAN, ROBERT J. MARSH, AND IDUN REITEN
}

\begin{abstract}
Associated to any acyclic cluster algebra is a corresponding triangulated category known as the cluster category. It is known that there is a one-to-one correspondence between cluster variables in the cluster algebra and exceptional indecomposable objects in the cluster category inducing a correspondence between clusters and cluster-tilting objects.

Fix a cluster-tilting object $T$ and a corresponding initial cluster. By the Laurent phenomenon, every cluster variable can be written as a Laurent polynomial in the initial cluster. We give conditions on $T$ equivalent to the fact that the denominator in the reduced form for every cluster variable in the cluster algebra has exponents given by the dimension vector of the corresponding module over the endomorphism algebra of $T$.
\end{abstract}

\section{INTRODUCTION}

Cluster algebras were introduced by Fomin-Zelevinsky [FZ1, and have been influential in many settings including algebraic combinatorics, Lie theory, Poisson geometry, Teichmüller theory, total positivity and quiver representations.

The first link to quiver representations and tilting theory for algebras was given in [MRZ]. Cluster categories were defined in [BMRRT], giving a categorical model for cluster combinatorics. For type $A$, an independent approach was given in CCS1.

A cluster algebra is in essence a commutative ring with a distinguished countable family of generators, called cluster variables. The cluster variables can be grouped into overlapping sets of equal finite size $n$, called clusters. Any given cluster variable can be uniquely expressed as a rational function in the elements of a fixed initial cluster. By the Laurent phenomenon, [FZ1, 3.1], these rational functions are actually Laurent polynomials with integer coefficients.

Corresponding to each cluster there is a quiver $Q$, with $n$ vertices. The cluster algebra is said to be acyclic if it admits a cluster for which the corresponding quiver $Q$ has no oriented cycles. In this case there is a corresponding finite dimensional hereditary algebra, the path algebra of $k Q$ (for some field $k$ ) giving rise to a cluster category.

In BMRT a surjective map $\alpha$ from the cluster variables of an acyclic cluster algebra to the exceptional indecomposable objects in the corresponding cluster category was given. In CK2 a map was defined in the opposite direction, and it was shown that this map is a bijection with $\alpha$ as inverse (see also [BCKMRT]). Our exposition is made in such a way that it is easy to see where the fact that $\alpha$ is a bijection is used.

An interesting property of the map $\alpha$ from BMRT is the following. Choose a cluster whose corresponding quiver is acyclic. If a cluster variable (not from this cluster) is expressed as a Laurent polynomial in the cluster variables from this

2000 Mathematics Subject Classification. Primary: 16G20, 16S99; Secondary 16G70, 16E99, 17B99.

Key words and phrases. Cluster algebra, cluster-tilted algebra, path algebra, cluster category, denominator, tilting theory.

The authors were supported by Storforsk grant no. 167130 from the Norwegian Research Council. 
cluster there is a natural interpretation of the monomial in the denominator in terms of the composition factors of its image under $\alpha$ (regarded as a $k Q$-module). This generalized earlier results in this direction [FZ2, CCS2, CK1, RT].

It is interesting to ask whether for an arbitrary choice of initial cluster it is possible to interpret the monomial in the denominator of a cluster variable in terms of the composition factors of the module (over an appropriate cluster-tilted algebra) corresponding to the image of the cluster variable under $\alpha$. The cluster-tilted algebra involved should be the endomorphism algebra of the cluster-tilting object $\tau T$ obtained by forming the direct sum of the images of the fixed initial cluster under $\alpha$. We remark that such an interpretation has been found in the Dynkin case in $\mathrm{CCS} 2, \mathrm{RT}$.

In this paper we give a precise answer to this question for the general case by giving necessary and sufficient conditions on the cluster-tilting object $T$ for this to hold. For a given acyclic cluster algebra, these conditions hold for all cluster-tilting objects $T$ in the corresponding cluster category if and only if the algebra is of finite cluster type or of rank 2 . In the tame case we show that a cluster-tilting object $T$ satisfies the conditions if and only if no indecomposable regular summand of $T$ has quasilength exactly one less than the rank of the tube in which it lies, and that this is equivalent to $\operatorname{End}_{\mathcal{C}}\left(T_{i}\right) \simeq k$ for each indecomposable summand of $T$. In fact this last condition is necessary for any path algebra $k Q$, and we conjecture that it is also sufficient in general.

We would like to thank Otto Kerner for answering several questions on wild algebras, and especially for assisting us with the proof of Proposition 2.6.

\section{BaCKGround And Main Results}

Let $Q$ be a finite connected acyclic quiver and $k$ an algebraically closed field. Let $H=k Q$ be the corresponding (finite dimensional) path algebra and $D^{b}(k Q)$ the bounded derived category of finite dimensional $k Q$-modules. It has autoequivalences $\tau$ (the Auslander-Reiten translate) and [1] (the shift). Let $\mathcal{C}=D^{b}(k Q) / \tau^{-1}[1]$ be the corresponding cluster category. We shall regard modules over $k Q$ as objects of $\mathcal{C}$ (via the natural embedding of the module category over $k Q$ in $D^{b}(k Q)$ ). For $i$ a vertex of $Q$ let $P_{i}$ denote the corresponding indecomposable projective $k Q$-module.

Let $\mathcal{A}=\mathcal{A}(Q) \subseteq \mathbb{F}=\mathbb{Q}\left(x_{1}, x_{2}, \ldots, x_{n}\right)$ be the (acyclic, coefficient-free) cluster algebra defined using the initial seed $(\mathbf{x}, Q)$, where $\mathbf{x}$ is the transcendence basis $\left\{x_{1}, x_{2}, \ldots, x_{n}\right\}$ of $\mathbb{F}$.

For an object $M$ of $\mathcal{C}$, let $c_{M}=\prod_{i=1}^{n} x_{i}^{\operatorname{dim} \operatorname{Hom}_{\mathcal{C}}\left(P_{i}, M\right)}$. For a polynomial $f=$ $f\left(z_{1}, z_{2}, \ldots, z_{n}\right)$, we say that $f$ satisfies the positivity condition if $f\left(e_{i}\right)>0$ where $e_{i}=(1, \ldots, 1,0,1, \ldots, 1)$ (with a 0 in the $i$ th position) for $i=1,2, \ldots, n$. This condition was crucial in the investigations in [BMRT, and also plays an essential role here.

We first recall the following results.

Theorem 1.1. BMRT, 2.2] Every cluster variable of $\mathcal{A}$ is either of the form $f / c_{M}$ (for some exceptional $k Q$-module $M$ ) or of the form $f x_{i}$ for some $i$, where in either case $f=f\left(x_{1}, x_{2}, \ldots, x_{n}\right)$ satisfies the positivity condition. Every indecomposable exceptional $k Q$-module arises in this way, and if $M$ and $M^{\prime}$ are two such modules satisfying $c_{M}=c_{M^{\prime}}$ then $M \simeq M^{\prime}$.

A map $\alpha$ is then defined from cluster variables of $\mathcal{A}$ to (isomorphism classes of) exceptional indecomposable objects of $\mathcal{C}$ in the following way. If $f / c_{M}$ is a cluster variable for some exceptional indecomposable $k Q$-module $M$ then $\alpha\left(f / c_{M}\right)=M$. If $f x_{i}$ is a cluster variable then $\alpha\left(f x_{i}\right)=P_{i}[1]$. 
Recall that a tilting seed for $\mathcal{A}$ is a pair $\left(T, Q^{\prime}\right)$ where $T$ is a cluster-tilting object of $\mathcal{C}$ and $Q^{\prime}$ is the quiver of $\operatorname{End}_{\mathcal{C}}(T)^{\mathrm{op}}$.

Theorem 1.2. BMRT, 2.3] The map

$\alpha:\{$ cluster variables of $\mathcal{A}(Q)\} \rightarrow\{$ indecomposable exceptional objects in $\mathcal{C}\}$ is surjective. It induces a surjective map $\bar{\alpha}:\{$ clusters $\} \rightarrow\{$ cluster-tilting objects $\}$, and a surjective map $\widetilde{\alpha}:\{$ seeds $\} \rightarrow\{$ tilting seeds $\}$, preserving quivers.

We also have the following result of [CK1], see also BCKMRT].

Theorem 1.3. There is a bijection $\beta: M \rightarrow X_{M}$ from indecomposable exceptional objects of $\mathcal{C}$ to cluster variables of $\mathcal{A}$ such that for any indecomposable exceptional $k Q$-module $M$, we have $X_{M}=f / c_{M}$ as an irreducible fraction of integral polynomials in the $x_{i}$.

In particular $\alpha$ is an inverse of $\beta$ and for the cluster variables $f x_{i}$ considered in Theorem 1.1, we can only have $f=1$.

Let $\Gamma$ be a quiver mutation-equivalent to $Q$. Then there is a seed $(\mathbf{y}, \Gamma)$ of $\mathcal{A}$, where $\mathbf{y}=\left\{y_{1}, y_{2}, \ldots, y_{n}\right\}$ is a transcendence basis of $\mathbb{F}$. For $i=1,2, \ldots, n$ let $T_{i}=\tau^{-1} \alpha\left(y_{i}\right)$ so that we have $\alpha\left(y_{i}\right)=\tau T_{i}$. It follows from Theorem 1.2 that $\amalg_{i=1}^{n} \tau T_{i}$ is a cluster-tilting object in $\mathcal{C}$ and that the quiver of its endomorphism algebra in $\mathcal{C}$ is $\Gamma$. Hence the same holds for $T=\amalg_{i=1}^{n} T_{i}$.

We can ask whether results analogous to the above hold for cluster variables when they are expressed in terms of the initial cluster $\left\{y_{1}, y_{2}, \ldots, y_{n}\right\}$. For $M$ an object in $\mathcal{C}$, let $t_{M}=\prod_{i=1}^{n} y_{i}^{\operatorname{dim} \operatorname{Hom}_{\mathcal{C}}\left(T_{i}, M\right)}$.

An interesting open question is whether $t_{M}$ (or, equivalently, the dimensions of the spaces $\operatorname{Hom}_{\mathcal{C}}\left(T_{i}, M\right)$ for $\left.i=1,2, \ldots, n\right)$ determines $M$ uniquely. This is true if $T=k Q$ is the direct sum of the non-isomorphic indecomposable projective modules but is not known in general. In particular, this means that an approach following [BMRT] exactly in this more general context is not possible.

Definition 1.4. Let $x$ be a cluster variable of $\mathcal{A}$. We say that $x$ expressed in terms of the cluster $\mathbf{y}$ has a $T$-denominator if either:

(I) We have that $\alpha(x)=M$ for some exceptional indecomposable object $M$ of $\mathcal{C}$ not isomorphic to $\tau T_{i}$ for any $i$, and $x=f / t_{M}$, or

(II) We have that $\alpha(x)=\tau T_{i}$ for some $i$ and $x=f y_{i}$.

In either case, $f$ is a polynomial in the $y_{i}$ satisfying the positivity condition.

Our first main result is the following:

Theorem 1.5. Let $Q$ be a finite quiver with no oriented cycles, and let $k$ be an algebraically closed field. Let $\mathcal{C}$ be the cluster category associated to $k Q$, and let $T=\amalg_{i=1}^{n} T_{i}$ be a cluster-tilting object in $\mathcal{C}$. Let $\mathcal{A}=\mathcal{A}(Q)$ be the cluster algebra associated to $Q$. Then:

(a) If no indecomposable direct summand of $T$ is regular then every cluster variable of $\mathcal{A}$ has a $T$-denominator.

(b) If every cluster variable of $\mathcal{A}$ has a $T$-denominator, then $\operatorname{End}_{\mathcal{C}}\left(T_{i}\right) \simeq k$ for all $i$.

We note that, as in Theorem 1.1, the bijectivity of $\alpha$ can be used to show that in case (II) of Definition 1.4, only $f=1$ occurs. The proof of Theorem 1.5] will be completed at the end of Section 4. In the special case where $k Q$ is a tame algebra, we have the following:

Theorem 1.6. Suppose that we are in the situation of Theorem 1.5 and that, in addition, $k Q$ is a tame algebra. Then the following are equivalent: 
(a) Every cluster variable of $\mathcal{A}$ has a $T$-denominator.

(b) No regular summand $T_{i}$ of quasi-length $r-1$ lies in a tube of rank $r$.

(c) For all $i, \operatorname{End}_{\mathcal{C}}\left(T_{i}\right) \simeq k$.

The proof of Theorem 1.6 will be completed at the end of Section 6. As a consequence of our main results, we have in the general case:

Corollary 1.7. Let $Q$ be a finite quiver with no oriented cycles. Let $\mathcal{C}$ be the cluster category associated to $k Q$. Let $\mathcal{A}=\mathcal{A}(Q)$ be the cluster algebra associated to $Q$. Then every cluster variable of $\mathcal{A}$ has a $T$-denominator for every cluster-tilting object $T$ if and only if $Q$ is Dynkin or has exactly two vertices.

The proof of Corollary 1.7 will be completed at the end of Section 4

\section{Conditions on a Cluster-tilting object}

In this section we consider certain conditions on a cluster-tilting object which are equivalent to all cluster variables in $\mathcal{A}$ having a $T$-denominator. Fix an almost complete (basic) cluster-tilting object $\bar{T}^{\prime}$ in $\mathcal{C}$. Let $M, M^{*}$ be the two complements of $\bar{T}^{\prime}$, so that $T^{\prime}=\bar{T}^{\prime} \amalg M$ and $T^{\prime \prime}=\bar{T}^{\prime} \amalg M^{*}$ are cluster-tilting objects (see BMRRT, $5.1])$. Let

$$
\begin{aligned}
& M^{*} \stackrel{f}{\rightarrow} B \stackrel{g}{\rightarrow} M \stackrel{h}{\rightarrow} \\
& M \stackrel{f^{\prime}}{\rightarrow} B^{\prime} \stackrel{g^{\prime}}{\rightarrow} M^{*} \stackrel{h^{\prime}}{\rightarrow}
\end{aligned}
$$

be the exchange triangles corresponding to $M$ and $M^{*}$ (see [BMRRT, §6]), so that $B \rightarrow M$ is a minimal right add $\left(\bar{T}^{\prime}\right)$-approximation of $M$ in $\mathcal{C}$ and $B^{\prime} \rightarrow M^{*}$ is a minimal right add $\left(\bar{T}^{\prime}\right)$-approximation of $M^{*}$ in $\mathcal{C}$. The following definition appears to be important for understanding the link between representation theory and denominators of cluster variables:

Definition 2.1. Let $N$ be an exceptional indecomposable object of $\mathcal{C}$. We say that $N$ is compatible with an exchange pair $\left(M, M^{*}\right)$ if the following holds whenever $M \nsucceq \tau N \not M^{*}$ :

$$
\begin{aligned}
\operatorname{dim} \operatorname{Hom}_{\mathcal{C}}(N, M)+\operatorname{dim} \operatorname{Hom}_{\mathcal{C}}\left(N, M^{*}\right) & \\
& =\max \left(\operatorname{dim} \operatorname{Hom}_{\mathcal{C}}(N, B), \operatorname{dim}_{\mathcal{C}} \operatorname{Hom}_{\mathcal{C}}\left(N, B^{\prime}\right)\right)
\end{aligned}
$$

If $N$ is compatible with every exchange pair $\left(M, M^{*}\right)$ in $\mathcal{C}$ we call $N$ exchange compatible.

We will investigate when this condition is satisfied. Note that the case when $M \simeq$ $\tau N$ or $M^{*} \simeq \tau N$ is covered by the following.

Lemma 2.2. Let $N$ be an exceptional indecomposable object of $\mathcal{C}$ and suppose that $M \simeq \tau N$ or $M^{*} \simeq \tau N$. Then we have that

$$
\begin{aligned}
& \operatorname{dim} \operatorname{Hom}_{\mathcal{C}}(N, M)+\operatorname{dim} \operatorname{Hom}_{\mathcal{C}}\left(N, M^{*}\right)= \\
& \qquad \max \left(\operatorname{dim} \operatorname{Hom}_{\mathcal{C}}(N, B), \operatorname{dim}_{\left.\operatorname{Hom}_{\mathcal{C}}\left(N, B^{\prime}\right)\right)+1}\right.
\end{aligned}
$$

Proof. Assume that $M^{*} \simeq \tau N$ (the other case is similar). Then there are exchange triangles $M \rightarrow B \rightarrow \tau N \rightarrow$ and $\tau N \rightarrow B^{\prime} \rightarrow M \rightarrow$. We have that $\operatorname{Hom}_{\mathcal{C}}(N, M) \simeq$ $D \operatorname{Ext}_{\mathcal{C}}^{1}(M, \tau N)$. This space is one-dimensional, since $(M, \tau N)$ is an exchange pair (by [BMRRT, 7.5]). Since $N$ is exceptional, $\operatorname{Hom}_{\mathcal{C}}(N, \tau N)=0$, and we have $\operatorname{Hom}_{\mathcal{C}}\left(N, B \amalg B^{\prime}\right) \simeq D \operatorname{Ext}^{1}\left(B \amalg B^{\prime}, \tau N\right)=0$, since $B \amalg B^{\prime} \amalg \tau N$ can be completed to a cluster-tilting object. 
Proposition 2.3. Suppose that neither $M$ nor $M^{*}$ is isomorphic to $\tau N$. Then the following are equivalent:

(a) We have that

$$
\begin{aligned}
\operatorname{dim} \operatorname{Hom}_{\mathcal{C}}(N, M)+\operatorname{dim} \operatorname{Hom}_{\mathcal{C}}\left(N, M^{*}\right) & \\
& =\max \left(\operatorname{dim} \operatorname{Hom}_{\mathcal{C}}(N, B), \operatorname{dim}_{\left.\operatorname{Hom}_{\mathcal{C}}\left(N, B^{\prime}\right)\right) .}\right.
\end{aligned}
$$

(b) Either the sequence

$$
0 \rightarrow \operatorname{Hom}_{\mathcal{C}}\left(N, M^{*}\right) \stackrel{a}{\rightarrow} \operatorname{Hom}_{\mathcal{C}}(N, B) \stackrel{b}{\rightarrow} \operatorname{Hom}_{\mathcal{C}}(N, M) \rightarrow 0
$$

is exact, or the sequence

$$
0 \rightarrow \operatorname{Hom}_{\mathcal{C}}(N, M) \stackrel{c}{\rightarrow} \operatorname{Hom}_{\mathcal{C}}\left(N, B^{\prime}\right) \stackrel{d}{\rightarrow} \operatorname{Hom}_{\mathcal{C}}\left(N, M^{*}\right) \rightarrow 0
$$

is exact.

Proof. Consider the (not necessarily exact) sequence:

$\operatorname{Hom}_{\mathcal{C}}\left(N, M^{*}\right) \stackrel{a}{\rightarrow} \operatorname{Hom}_{\mathcal{C}}(N, B) \stackrel{b}{\rightarrow} \operatorname{Hom}_{\mathcal{C}}(N, M) \stackrel{c}{\rightarrow} \operatorname{Hom}_{\mathcal{C}}\left(N, B^{\prime}\right) \stackrel{d}{\rightarrow} \operatorname{Hom}_{\mathcal{C}}\left(N, M^{*}\right)$

By exactness at the second term we have that $\operatorname{Ker} b=\operatorname{Im} a$. Let $r=\operatorname{dim} \operatorname{Hom}_{\mathcal{C}}\left(N, M^{*}\right)$, $s=\operatorname{dim} \operatorname{Hom}_{\mathcal{C}}(N, B), t=\operatorname{dim} \operatorname{Hom}_{\mathcal{C}}(N, M)$ and $u=\operatorname{dim}_{\operatorname{Hom}}\left(N, B^{\prime}\right)$. Then we have that $s=\operatorname{dim} \operatorname{Ker} b+\operatorname{dim} \operatorname{Im} b=\operatorname{dim} \operatorname{Im} a+\operatorname{dim} \operatorname{Im} b \leq r+t$. Similarly, by exactness at the fourth term, we have that $u \leq t+r$.

Then the sequence (3) is exact if and only if $b$ is surjective and $a$ is injective, if and only if $\operatorname{dim} \operatorname{Im} b=t$ and $\operatorname{dim} \operatorname{Im} a=r$. By the above argument that $s \leq r+t$, we see that this is true if and only if $s=r+t$. Similarly, the sequence (44) is exact if and only if $u=r+t$. Hence (b) holds for $N$ if and only if $s=r+t$ or $u=r+t$. Since in any case $s \leq r+t$ and $u \leq r+t$, we see that (b) holds if and only $r+t=\max (s, u)$ as required.

Let $X, Y$ be indecomposable objects of $\mathcal{C}$. We can always represent such objects by indecomposable $k Q$-modules or objects of the form $P_{i}[1]$ for some $i$. Call these also $X$ and $Y$, by abuse of notation. By [BMRRT, 1.5], $\operatorname{Hom}_{\mathcal{C}}(X, Y)=$ $\operatorname{Hom}_{\mathcal{D}}(X, Y) \amalg \operatorname{Hom}_{\mathcal{D}}(X, F Y)$. We call an element of $\operatorname{Hom}_{H}(X, Y)$ an $H$-map from $X$ to $Y$, and an element of $\operatorname{Hom}_{\mathcal{D}}(X, F Y)$ an $F$-map from $X$ to $Y$. We note that the composition of two $H$-maps is an $H$-map and the composition of two $F$-maps is zero. The composition of an $H$-map and an $F$-map is an $F$-map.

Proposition 2.4. If $N$ is an indecomposable exceptional object with $\operatorname{End}_{\mathcal{C}}(N) \nsucceq$ $k$, then there is an indecomposable exceptional object $N^{*}$ such that $\left(N, N^{*}\right)$ is an exchange pair and $N$ is not compatible with this exchange pair.

Proof. Let $E$ be the Bongartz complement of $N$, as defined in $[\mathrm{B}$. Then $N \amalg E$ is a tilting module such that $\operatorname{Hom}_{H}(N, E)=0$ (see [H]).

Now consider $N \amalg E$ as a cluster-tilting object in $\mathcal{C}$, and consider the exchange triangles $N \rightarrow B^{\prime} \rightarrow N^{*}$ and $N^{*} \rightarrow B \rightarrow N$, where $B$ and $B^{\prime}$ are in add $E$.

Then the map $N \rightarrow B^{\prime}$ is a sum of $F$-maps. Since by assumption $\operatorname{End}_{\mathcal{C}}(N) \nsucceq k$, we have a non-zero $F$-map $N \stackrel{\epsilon}{\rightarrow} N$. The composition $N \stackrel{\epsilon}{\rightarrow} N \rightarrow B^{\prime}$ is zero, as the composition of $F$-maps is zero. So $\operatorname{Hom}_{\mathcal{C}}(N$,$) applied to N \rightarrow B^{\prime} \rightarrow N^{*}$ does not give a short exact sequence.

It is clear that $\operatorname{Hom}_{\mathcal{C}}(N$,$) applied to N^{*} \rightarrow B \rightarrow N$ does not give a short exact sequence, since the identity map $N \rightarrow N$ does not factor through $B \rightarrow N$.

We claim that $N$ is not compatible with $\left(N, N^{*}\right)$. We have that $\operatorname{Ext}_{\mathcal{C}}^{1}(N, \tau N) \simeq$ $D \operatorname{Hom}_{\mathcal{C}}(N, N) \not k$, so $(N, \tau N)$ is not an exchange pair by [BMRRT, 7.5]. It follows that $N^{*} \nsucceq \tau N$. We also have that $N \nsucceq \tau N$, since $N$ is exceptional. So we are done by combining the above with Proposition 2.3 . 


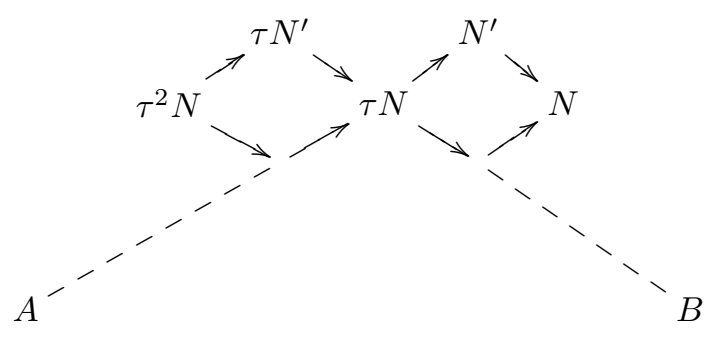

Figure 1. The proof of Proposition 2.6

We have the following immediate consequence.

Corollary 2.5. If $N$ is an exchange compatible object of $\mathcal{C}$, then $\operatorname{End}_{\mathcal{C}}(N) \simeq k$.

There is the following description of the indecomposable exceptional modules $N$ with $\operatorname{End}_{\mathcal{C}}(N) \nsucceq k$. We know that such $N$ must be regular. In the tame case the indecomposable exceptional modules in a tube of rank $t$ are those of quasilength at most $t-1$. In the wild case, for a component of the AR-quiver there is some $r$ with $0 \leq r \leq n-2$, where $n$ is the number of simple modules, such that $N$ is exceptional if and only if $N$ has quasilength at most $r$.

Let $X$ be an indecomposable regular module. Then we denote by $\Delta_{X}$ the wing of $X$, as defined in [R1, 3.3].

The proof of the next result, which is based on the proof of [K1, 1.6], [K2, 9.5], was pointed out to us by Kerner.

Proposition 2.6. Assume that $H$ is hereditary of infinite type, and that $N$ is an indecomposable exceptional regular $H$-module. Then $\operatorname{End}_{\mathcal{C}}(N) \not z k$ if and only if $N$ has maximal quasilength amongst the indecomposable exceptional modules in its component.

Proof. Let $N$ be indecomposable exceptional regular of quasilength $t$, and let $N^{\prime}$ be indecomposable with an irreducible epimorphism $N \rightarrow N^{\prime}$. Then $\operatorname{End}_{\mathcal{C}}(N) \not z k$ if and only if $\operatorname{Hom}_{H}\left(N, \tau^{2} N\right) \neq 0$, and $N^{\prime}$ is exceptional if and only if $\operatorname{Hom}_{H}\left(N^{\prime}, \tau N^{\prime}\right) \simeq$ $\operatorname{Ext}_{H}^{1}\left(N^{\prime}, N^{\prime}\right)=0$, if and only if $N$ does not have maximal quasilength amongst the indecomposable exceptional modules in its component (note that all indecomposable modules in a $\tau$-orbit of an AR-component of a hereditary algebra are always either all exceptional or all not exceptional).

Consider the exact sequences $0 \rightarrow A \rightarrow N^{\prime} \rightarrow N \rightarrow 0$ and $0 \rightarrow \tau^{2} N \rightarrow \tau N^{\prime} \rightarrow$ $B \rightarrow 0$. Since $N^{\prime} \rightarrow N$ is an epimorphism and $\tau^{2} N \rightarrow \tau N^{\prime}$ is a monomorphism, it follows that $\operatorname{Hom}_{H}\left(N, \tau^{2} N\right) \neq 0$ implies $\operatorname{Hom}_{H}\left(N^{\prime}, \tau N^{\prime}\right) \neq 0$.

Assume conversely that there is a nonzero map $f: N^{\prime} \rightarrow \tau N^{\prime}$. Since the wing $\Delta_{\tau N^{\prime}}$ determined by $\tau N^{\prime}$ is standard $\mathrm{K} 2$, we have that $\operatorname{Hom}\left(A, \tau N^{\prime}\right)=0$ (see Figure 1). Hence the composition of the map $A \rightarrow N^{\prime}$ with $N \rightarrow \tau N^{\prime}$ is zero. It follows that the map $N^{\prime} \rightarrow \tau N^{\prime}$ factors through $N$ and therefore that there is a non-zero map $f^{\prime}: N \rightarrow \tau N^{\prime}$. Similarly, considering the wing $\Delta_{N^{\prime}}$, we see that $\operatorname{Hom}(N, B)=0$. Hence the composition of $f^{\prime}$ with the map $\tau N^{\prime} \rightarrow B$ is zero, and $f^{\prime}$ factors through $\tau^{2} N$ and therefore there is a non-zero map $N \rightarrow \tau^{2} N$. Hence we see that $\operatorname{End}_{\mathcal{C}}(N) \not k$ if and only if $N$ is exceptional of maximal quasilength.

Remark 2.7. The proof in the tame case can be made even shorter.

We note the following consequence of Lemma 2.5 and Proposition 2.6. 
Corollary 2.8. Assume that $H$ is of infinite type. If $N$ is indecomposable regular and has maximal quasilength amongst the indecomposable exceptional modules in its component, then $N$ is not exchange compatible.

\section{EXPRESSIONS FOR CLUSTER VARIABLES}

In this section we show that for a given cluster-tilting object $T$ in the cluster category of $k Q$, all indecomposable direct summands $T_{j}$ of $T$ are exchange compatible if and only if all cluster variables in $\mathcal{A}(Q)$ have $T$-denominators.

Let $\left(\mathbf{x}^{\prime}, Q^{\prime}\right)$ be a seed, with $\mathbf{x}=\left\{x_{1}^{\prime}, \ldots, x_{n}^{\prime}\right\}$, and assume that each $x_{i}^{\prime}$ has a $T$-denominator. Let $T_{i}^{\prime}=\alpha\left(x_{i}^{\prime}\right)$ for $i=1,2, \ldots, n$ and set $T^{\prime}=\amalg_{j=1}^{n} T_{j}^{\prime}$. By Theorem 1.2, we have that $T^{\prime}$ is a cluster-tilting object in $\mathcal{C}$ and that $Q^{\prime}$ is the quiver of $\operatorname{End}_{\mathcal{C}}\left(T^{\prime}\right)^{\text {op }}$.

Mutating $\left(\mathbf{x}^{\prime}, Q^{\prime}\right)$ at $x_{k}^{\prime}$ we obtain a new cluster variable $\left(x_{k}^{\prime}\right)^{*}$. Let $\bar{T}^{\prime}=\amalg_{j \neq k} T_{j}^{\prime}$. Let $\left(T_{k}^{\prime}\right)^{*}$ be the unique indecomposable object in $\mathcal{C}$ with $\left(T_{k}^{\prime}\right)^{*} \nsucceq T_{k}^{\prime}$ such that $\bar{T}^{\prime} \amalg\left(T_{k}^{\prime}\right)^{*}$ is a cluster-tilting object.

Proposition 3.1. With the above notation and assumptions, the cluster variable $\left(x_{k}^{\prime}\right)^{*}$ has a $T$-denominator if and only if each summand $T_{i}$ of $T$ is compatible with the exchange pair $\left(T_{k}^{\prime},\left(T_{k}^{\prime}\right)^{*}\right)$.

Proof. We consider the exchange triangles corresponding to the almost complete cluster-tilting object $\bar{T}^{\prime}$ in $\mathcal{C}$ (see triangles (11) and (2)). For an indecomposable direct summand $A$ of $T^{\prime}$, we denote the corresponding cluster variable in $\mathrm{x}^{\prime}$ by $x_{A}$. Note that all summands of $B$ and $B^{\prime}$ are also summands of $\bar{T}^{\prime}$. We therefore have, by assumption, that for any summand $A$ of $B$ or $B^{\prime}$ (or $A=T_{k}^{\prime}$ ), either $A \not \tau T_{i}$ for any $i$, and $x_{A}=f_{A} / t_{A}$, or $A \simeq \tau T_{i}$ for some $i$ and $x_{A}=f_{A} y_{i}$, where in either case $f_{A}$ is a polynomial satisfying the positivity condition. We also define $M=T_{k}^{\prime}$ and $M^{*}=\left(T_{k}^{\prime}\right)^{*}$. We note that by BMRT, 3.1], $\alpha\left(\left(x_{k}^{\prime}\right)^{*}\right)=M^{*}$.

If $A_{1}, A_{2}, \ldots, A_{t}$ are summands of $T^{\prime}$, we write

$$
x_{A_{1} \amalg A_{2} \amalg \cdots \amalg A_{t}}=x_{A_{1}} x_{A_{2}} \cdots x_{A_{t}}
$$

and

$$
f_{A_{1} \amalg A_{2} \amalg \cdots \amalg A_{t}}=f_{A_{1}} f_{A_{2}} \cdots f_{A_{t}} .
$$

We also write

$$
y_{\tau T_{i_{1}} \amalg \tau T_{i_{2}} \amalg \cdots \amalg \tau T_{i_{t}}}=y_{i_{1}} y_{i_{2}} \cdots y_{i_{t}}
$$

for any $i_{1}, i_{2}, \ldots, i_{t} \in\{1,2, \ldots, n\}$.

We have BMR2] that

$$
x_{M}\left(x_{M}\right)^{*}=x_{B}+x_{B^{\prime}},
$$

The argument now falls into different cases.

Case (I):

Suppose first that neither $M$ nor $M^{*}$ is isomorphic to $\tau T_{i}$ for any $i$. Write $B=$ $B_{0} \amalg B_{1}$, where no summand of $B_{0}$ is of the form $\tau T_{i}$ for any $i$. Similarly, write $B^{\prime}=B_{0}^{\prime} \amalg B_{1}^{\prime}$, where no summand of $B_{0}^{\prime}$ is of the form $\tau T_{i}$ for any $i$. Then by assumption $x_{B}=\frac{f_{B} y_{B_{1}}}{t_{B_{0}}}$. Similarly $x_{B^{\prime}}=\frac{f_{B^{\prime}} y_{B_{1}^{\prime}}}{t_{B_{0}^{\prime}}}$. 
Let $m=\frac{\operatorname{lcm}\left(t_{B_{0}}, t_{B_{0}^{\prime}}\right)}{t_{B_{0}}}$ and $m^{\prime}=\frac{\operatorname{lcm}\left(t_{B_{0}}, t_{B_{0}^{\prime}}\right)}{t_{B_{0}^{\prime}}}$. We have:

$$
\begin{aligned}
\left(x_{M}\right)^{*} & =\frac{\frac{f_{B} y_{B_{1}}}{t_{B_{0}}}+\frac{f_{B^{\prime}} y_{B_{1}^{\prime}}}{t_{B_{0}^{\prime}}}}{\frac{f_{M}}{t_{M}}} \\
& =\frac{f_{B} m y_{B_{1}}+f_{B^{\prime}} m^{\prime} y_{B_{1}^{\prime}}}{\operatorname{lcm}\left(t_{B_{0}}, t_{B_{0}^{\prime}}\right) f_{M} / t_{M}} \\
& =\frac{\left(f_{B} m y_{B_{1}}+f_{B^{\prime}} m^{\prime} y_{B_{1}^{\prime}}\right) / f_{M}}{\operatorname{lcm}\left(t_{B}, t_{B^{\prime}}\right) / t_{M}}
\end{aligned}
$$

noting that $t_{B}=t_{B_{0}}$ since $\operatorname{Hom}_{\mathcal{C}}\left(T_{i}, \tau T_{j}\right)=0$ for all $i, j$, and similarly $t_{B^{\prime}}=t_{B_{0}^{\prime}}$.

Assume now that each of the $T_{i}$ is compatible with $\left(M, M^{*}\right)$, so that we have

$$
\begin{aligned}
& t_{M} t_{M^{*}}=\prod y_{i}^{\operatorname{dim} \operatorname{Hom}_{\mathcal{C}}\left(T_{i}, M\right)+\operatorname{dim} \operatorname{Hom}_{\mathcal{C}}\left(T_{i}, M^{*}\right)} \\
& =\prod y_{i}^{\max \left(\operatorname{dim} \operatorname{Hom}_{\mathcal{C}}(N, B), \operatorname{dim} \operatorname{Hom}_{\mathcal{C}}\left(N, B^{\prime}\right)\right)}=\operatorname{lcm}\left(t_{B}, t_{B^{\prime}}\right) .
\end{aligned}
$$

Hence

$$
\left(x_{M}\right)^{*}=\frac{\left(f_{B} m y_{B_{1}}+f_{B^{\prime}} m^{\prime} y_{B_{1}^{\prime}}\right) / f_{M}}{t_{M^{*}}} .
$$

By definition of least common multiple, $m$ and $m^{\prime}$ are coprime. Since $B$ and $B^{\prime}$ have no common factors [BMR2, 6.1], $y_{B_{1}}$ and $y_{B_{1}^{\prime}}$ are coprime. Suppose that $m$ and $y_{B_{1}^{\prime}}$ had a common factor $y_{i}$. Then there would be a summand $X$ of $B_{0}^{\prime}$ such that $\operatorname{Hom}_{\mathcal{C}}\left(T_{i}, X\right) \neq 0$, and $\tau T_{i}$ was a summand of $B^{\prime}$. But then

$$
\operatorname{Ext}_{\mathcal{C}}^{1}\left(X, \tau T_{i}\right) \simeq D \operatorname{Hom}_{\mathcal{C}}\left(\tau T_{i}, \tau X\right) \simeq D \operatorname{Hom}_{\mathcal{C}}\left(T_{i}, X\right) \neq 0,
$$

contradicting the fact that $B$ is the direct sum of summands of a cluster-tilting object. Therefore $m$ and $y_{B_{1}^{\prime}}$ are coprime, and similarly $m^{\prime}$ and $y_{B_{1}}$ are coprime. It follows that $m y_{B_{1}}$ and $m^{\prime} y_{B_{1}^{\prime}}$ are coprime.

Let $i \in\{1,2, \ldots, n\}$. It follows from our assumptions that $f_{B}\left(e_{i}\right)>0$ and $f_{B^{\prime}}\left(e_{i}\right)>0$. It is clear that $\left(m y_{B_{1}}\right)\left(e_{i}\right) \geq 0$ and $\left(m^{\prime} y_{B_{1}^{\prime}}\right)\left(e_{i}\right) \geq 0$. Since $m y_{B_{1}}$ and $m^{\prime} y_{B_{1}^{\prime}}$ are coprime, these two numbers cannot be simultaneously zero, so $\left(f_{B} m y_{B_{1}}+\right.$ $\left.f_{B^{\prime}} m^{\prime} y_{B_{1}^{\prime}}\right)\left(e_{i}\right)>0$. It follows that $f_{B} m y_{B_{1}}+f_{B^{\prime}} m^{\prime} y_{B_{1}^{\prime}}$ satisfies the positivity condition. By assumption, $f_{M}$ also satisfies the positivity condition.

Since $\left(x_{M}\right)^{*}$ is a Laurent polynomial in $y_{1}, y_{2}, \ldots, y_{n}$ by the Laurent phenomenon [FZ1, 3.1], $\left(f_{B} m y_{B_{1}}+f_{B^{\prime}} m^{\prime} y_{B_{1}^{\prime}}\right) / f_{M}$ is also a Laurent polynomial. Since it is defined at $e_{i}$ for all $i$, it must be a polynomial. By the above, it satisfies the positivity condition. It follows that $\left(x_{M}\right)^{*}$ has a $T$-denominator.

Now assume that at least one summand of $T$ is not compatible with $\left(M, M^{*}\right)$. Then $t_{M} t_{M^{*}}=c \operatorname{lcm}\left(t_{B}, t_{B^{\prime}}\right)$ for some nontrivial Laurent monomial $c$. We then obtain that

$$
\begin{aligned}
x_{M^{*}} & =\frac{\left(f_{B} m y_{B_{1}}+f_{B^{\prime}} m^{\prime} y_{B_{1}^{\prime}}\right) / f_{M}}{\operatorname{lcm}\left(t_{B}, t_{B^{\prime}}\right) / t_{M}} \\
& =c \cdot \frac{\left(f_{B} m y_{B_{1}}+f_{B^{\prime}} m^{\prime} y_{B_{1}^{\prime}}\right) / f_{M}}{t_{M^{*}}},
\end{aligned}
$$

where, as before, $\left(f_{B} m y_{B_{1}}+f_{B^{\prime}} m^{\prime} y_{B_{1}^{\prime}}\right) / f_{M}$ satisfies the positivity condition. It follows that the numerator $c \cdot\left(f_{B} m y_{B_{1}}+f_{B^{\prime}} m^{\prime} y_{B_{1}^{\prime}}\right) / f_{M}$ is either not a polynomial or does not satisfy the positivity condition and thus that $x_{M^{*}}$ does not have a $T$-denominator.

\section{Case (II):}

Next, suppose that $M \simeq \tau T_{i}$ for some $i$. Since $\operatorname{Ext}_{\mathcal{C}}^{1}\left(T_{r}, T_{s}\right)=0$ for all $r, s$, we

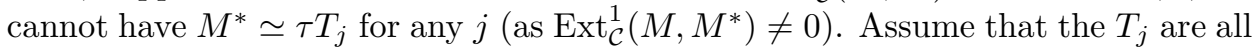


compatible with the exchange pair $\left(M, M^{*}\right)$. Then we have $t_{M^{*}}=y_{i} \operatorname{lcm}\left(t_{B}, t_{B^{\prime}}\right)$, noting that $t_{M}=1$. Here we use, in particular, that

$$
\begin{aligned}
\operatorname{dim} \operatorname{Hom}_{\mathcal{C}}(N, M)+\operatorname{dim} \operatorname{Hom}_{\mathcal{C}}\left(N, M^{*}\right) \\
=\max \left(\operatorname{dim} \operatorname{Hom}_{\mathcal{C}}(N, B), \operatorname{dim}_{\left.\operatorname{Hom}_{\mathcal{C}}\left(N, B^{\prime}\right)\right)+1}\right.
\end{aligned}
$$

holds for $N=T_{i}$, by Lemma 2.2. As in the above case, we have $x_{M}\left(x_{M}\right)^{*}=$ $x_{B}+x_{B^{\prime}}$. The same analysis, together with the fact that $x_{M}=f_{M} y_{i}$ for some polynomial $f$ in the $y_{j}$ satisfying the positivity condition, provides:

$$
\begin{aligned}
\left(x_{M}\right)^{*} & =\frac{\left(f_{B} m y_{B_{1}}+f_{B^{\prime}} m^{\prime} y_{B_{1}^{\prime}}\right) / f_{M}}{\operatorname{lcm}\left(t_{B}, t_{B^{\prime}}\right) y_{i}} \\
& =\frac{\left(f_{B} m y_{B_{1}}+f_{B^{\prime}} m^{\prime} y_{B_{1}^{\prime}}\right) / f_{M}}{t_{M^{*}}} .
\end{aligned}
$$

As above, we see that the numerator is a polynomial which satisfies the positivity condition, and it follows that $\left(x_{M}\right)^{*}$ has a $T$-denominator.

Now assume that at least one summand of $T$ is not compatible with $\left(M, M^{*}\right)$. Summands $T_{k} \nsucceq T_{i}$ which are not compatible with $\left(M, M^{*}\right)$, will not satisfy $\operatorname{dim} \operatorname{Hom}_{\mathcal{C}}(N, M)+\operatorname{dim} \operatorname{Hom}_{\mathcal{C}}\left(N, M^{*}\right)=\max \left(\operatorname{dim} \operatorname{Hom}_{\mathcal{C}}(N, B), \operatorname{dim} \operatorname{Hom}_{\mathcal{C}}\left(N, B^{\prime}\right)\right)$ for $N=T_{k}$.

It follows that $t_{M^{*}}=c y_{i} \operatorname{lcm}\left(t_{B}, t_{B^{\prime}}\right)$ for some nontrivial Laurent monomial $c$. Arguing as above we obtain that

$$
\begin{aligned}
x_{M^{*}} & =\frac{f_{B} m y_{B_{1}}+f_{B^{\prime}} m^{\prime} y_{B_{1}^{\prime}}}{t_{M^{*}} / c} \\
& =\left(\frac{f_{B} m y_{B_{1}}+f_{B^{\prime}} m^{\prime} y_{B_{1}^{\prime}}}{t_{M^{*}}}\right) c .
\end{aligned}
$$

Since $f_{B} m y_{B_{1}}+f_{B^{\prime}} m^{\prime} y_{B_{1}^{\prime}}$ satisfies the positivity condition, it follows that the numerator $\left(f_{B} m y_{B_{1}}+f_{B^{\prime}} m^{\prime} y_{B_{1}^{\prime}}\right) c$ is either not a polynomial or does not satisfy the positivity condition and therefore that $\left(x_{M}\right)^{*}$ does not have a $T$-denominator.

Case (III):

Next, suppose that $M^{*} \simeq \tau T_{i}$ for some $i$. Since $\operatorname{Ext}_{\mathcal{C}}^{1}\left(T_{r}, T_{s}\right)=0$ for all $r, s$,

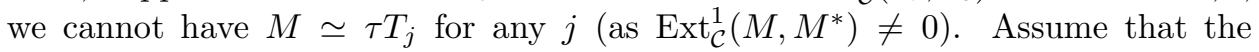
$T_{j}$ are all exchange compatible with $\left(M, M^{*}\right)$. We have, using Proposition [2.3. that $t_{M}=y_{i} \operatorname{lcm}\left(t_{B}, t_{B^{\prime}}\right)$, noting that $t_{M^{*}}=1$. As in the above case, we have $x_{M}\left(x_{M}\right)^{*}=x_{B}+x_{B^{\prime}}$. The same analysis provides:

$$
\begin{aligned}
\left(x_{M}\right)^{*} & =\frac{\left(f_{B} m y_{B_{1}}+f_{B^{\prime}} m^{\prime} y_{B_{1}^{\prime}}\right) / f_{M}}{\operatorname{lcm}\left(t_{B}, t_{B^{\prime}}\right) / t_{M}} \\
& =\left(\left(f_{B} m y_{B_{1}}+f_{B^{\prime}} m^{\prime} y_{B_{1}^{\prime}}\right) / f_{M}\right) y_{i} .
\end{aligned}
$$

As above, we see that $\left(f_{B} m y_{B_{1}}+f_{B^{\prime}} m^{\prime} y_{B_{1}^{\prime}}\right) / f_{M}$ is a polynomial which satisfies the positivity condition and it follows that $\left(x_{M}\right)^{*}$ has a $T$-denominator.

Now assume that at least one summand of $T$ is not compatible with $\left(M, M^{*}\right)$. Then, as in Case (II), it follows that $t_{M}=c y_{i} \operatorname{lcm}\left(t_{B}, t_{B^{\prime}}\right)$ for some nontrivial Laurent monomial $c$. Arguing as above we obtain that

$$
\begin{aligned}
\left(x_{M}\right)^{*} & =\frac{\left(f_{B} m y_{B_{1}}+f_{B^{\prime}} m^{\prime} y_{B_{1}^{\prime}}\right) / f_{M}}{\operatorname{lcm}\left(t_{B}, t_{B^{\prime}}\right) / t_{M}} \\
& =\left(\left(f_{B} m y_{B_{1}}+f_{B^{\prime}} m^{\prime} y_{B_{1}^{\prime}}\right) / f_{M}\right) c y_{i} .
\end{aligned}
$$

Since $f_{B} m y_{B_{1}}+f_{B^{\prime}} m^{\prime} y_{B_{1}^{\prime}}$ satisfies the positivity condition, it follows that $\left(f_{B} m y_{B_{1}}+\right.$ $\left.f_{B^{\prime}} m^{\prime} y_{B_{1}^{\prime}}\right) c$ is either not a polynomial or does not satisfy the positivity condition and therefore that $\left(x_{M}\right)^{*}$ does not have a $T$-denominator. 
Theorem 3.2. All indecomposable direct summands $T_{j}$ of $T$ are exchange compatible if and only if all cluster variables in $\mathcal{A}(Q)$ have a $T$-denominator.

Proof. We note that $\alpha\left(y_{i}\right)=\tau T_{i}$ for $i=1,2, \ldots, n$ and that the quiver $\Gamma$ of the seed $(\mathbf{y}, \Gamma)$ is the quiver of $\operatorname{End}_{\mathcal{C}}\left(\amalg_{j=1}^{n} \tau T_{j}\right)^{\text {op }}$ (see the start of Section 1). Suppose first that all indecomposable direct summands $T_{j}$ of $T$ are exchange compatible. Let $x$ be a cluster variable of $\mathcal{A}(Q)$. It follows from Proposition 3.1 by induction on the smallest number of exchanges needed to get from $\mathbf{y}$ to a cluster containing $x$ that $x$ has a $T$-denominator.

Suppose that there is an indecomposable direct summand of $T$ which is not exchange compatible. Consider the cluster $\mathbf{z}$ of $\mathcal{A}(Q)$ which is a minimal distance in the exchange graph from $\mathbf{y}$ such that some summand of $T$ is not compatible with an exchange pair $\left(\alpha(x), \alpha\left(x^{*}\right)\right)$ of modules coming from an exchange pair $\left(x, x^{*}\right)$ of cluster variables with $x \in \mathbf{z}$. Arguing as above, we see that the cluster variables in $\mathbf{z}$ all have a $T$-denominator. By Proposition 3.1 we see that $x^{*}$ does not have a $T$-denominator.

The fact that $\alpha$ is a bijection gives us more information, since it follows that $\alpha(x)=\tau T_{i}$ if and only if $x=y_{i}$. For the rest of this article, we freely use this fact. We therefore have:

Corollary 3.3. All indecomposable direct summands $T_{j}$ of $T$ are exchange compatible if and only if for every cluster variable $x$ of $\mathcal{A}(Q)$ we have:

(a) The object $\alpha(x)$ is not isomorphic to $\tau T_{i}$ for any $i$, and $x=f / t_{\alpha(x)}$ for some polynomial $f$ in the $y_{i}$ satisfying the positivity condition, or

(b) We have that $\alpha(x) \simeq \tau T_{i}$ for some $i$ and $x=y_{i}$.

Remark 3.4. Recall that the exponent of $y_{i}$ in $t_{M}$ is $d_{i}=\operatorname{dim} \operatorname{Hom}_{\mathcal{C}}\left(T_{i}, M\right)$. It follows from [BMR1, Theorem A] that $d_{i}$ is the multiplicity of the simple top of the projective $\Lambda=\operatorname{End}_{\mathcal{C}}(T)^{\mathrm{op}}$-module $\operatorname{Hom}_{\mathcal{C}}\left(T, T_{i}\right)$ as a composition factor in $\operatorname{Hom}_{\mathcal{C}}(T, M)$ : we have that

$$
\begin{aligned}
\operatorname{Hom}_{\mathcal{C}}\left(T_{i}, M\right) & \simeq \operatorname{Hom}_{\mathcal{C} / \operatorname{add}(\tau T)}\left(T_{i}, M\right) \\
& \simeq \operatorname{Hom}_{\Lambda}\left(\operatorname{Hom}_{\mathcal{C}}\left(T, T_{i}\right), \operatorname{Hom}_{\mathcal{C}}(T, M)\right),
\end{aligned}
$$

using the fact that $\operatorname{Hom}_{\mathcal{C}}\left(T_{i}, \tau T\right)=0$ (as $T$ is a cluster-tilting object in $\mathcal{C}$ ). Thus $\left(d_{1}, d_{2}, \ldots, d_{n}\right)$ is the dimension vector of the $\Lambda$-module $\operatorname{Hom}_{\mathcal{C}}(T, M)$.

\section{THE PREPROJECTIVE CASE}

In this section we adopt the notation from the previous section, but assume in addition that $N$ is a preprojective module, a preinjective module, or the shift of a projective module. Our aim is to show that such modules are exchange compatible.

Proposition 4.1. Suppose that neither $M$ nor $M^{*}$ is isomorphic to $\tau N$. Then at least one of the following holds (where the maps are induced from triangle (1) or triangle (2) respectively):

(a) The sequence

$$
0 \rightarrow \operatorname{Hom}_{\mathcal{C}}\left(N, M^{*}\right) \stackrel{a}{\rightarrow} \operatorname{Hom}_{\mathcal{C}}(N, B) \stackrel{b}{\rightarrow} \operatorname{Hom}_{\mathcal{C}}(N, M) \rightarrow 0
$$

is exact, or

(b) The sequence

$$
0 \rightarrow \operatorname{Hom}_{\mathcal{C}}(N, M) \stackrel{c}{\rightarrow} \operatorname{Hom}_{\mathcal{C}}\left(N, B^{\prime}\right) \stackrel{d}{\rightarrow} \operatorname{Hom}_{\mathcal{C}}\left(N, M^{*}\right) \rightarrow 0
$$

is exact. 
Proof. Assume first that $N$ is projective.

Case (I):

Suppose that $M$ and $M^{*}$ are both modules. By [BMRRT], we know that either triangle (11) or triangle (2) arises from a short exact sequence of modules. Suppose first that triangle (10) arises from a short exact sequence

$$
0 \rightarrow M^{*} \rightarrow B \rightarrow M \rightarrow 0
$$

of modules. Applying the exact functor $\operatorname{Hom}_{H}(N,-)$ to this, we obtain the exact sequence:

$$
0 \rightarrow \operatorname{Hom}_{H}\left(N, M^{*}\right) \rightarrow \operatorname{Hom}_{H}(N, B) \rightarrow \operatorname{Hom}_{H}(N, M) \rightarrow 0,
$$

noting that $\operatorname{Ext}_{H}^{1}\left(N, M^{*}\right)=0$ as $N$ is projective. $\operatorname{Since} \operatorname{Hom}_{\mathcal{C}}(N, X) \simeq \operatorname{Hom}_{H}(N, X)$ for all modules $X$ (cf. BMRRT, 1.7(d)]), we obtain (a) above. Similarly, if triangle (2) arises from a short exact sequence of modules, we obtain (b) above.

Case (II):

Suppose that $M=P[1]$ is the shift of a projective indecomposable module $P$. Since $\operatorname{Ext}_{\mathcal{C}}^{1}\left(P[1], P^{\prime}[1]\right)=0$ for any projective module $P^{\prime}$, we must have that $M^{*}$ is a module. Let $I$ be the indecomposable injective module corresponding to $P$. Since $H$ is the path algebra of an acyclic quiver, either $\operatorname{Hom}_{\mathcal{C}}(N, P)=\operatorname{Hom}_{H}(N, P)=0$ or $\operatorname{Hom}_{\mathcal{C}}(N, I)=\operatorname{Hom}_{H}(N, I)=0$. If $\operatorname{Hom}_{\mathcal{C}}(N, P)=0$, then, $\operatorname{applying} \operatorname{Hom}_{\mathcal{C}}(N$, to triangle (1), we obtain the exact sequence

$$
0 \rightarrow \operatorname{Hom}_{\mathcal{C}}\left(N, M^{*}\right) \rightarrow \operatorname{Hom}_{\mathcal{C}}(N, B) \rightarrow 0,
$$

noting that $\operatorname{Hom}_{\mathcal{C}}(N, M)=\operatorname{Hom}_{\mathcal{C}}(N, P[1])=0$ as $N$ is projective. We see that (a) follows.

If $\operatorname{Hom}_{\mathcal{C}}(N, I)=0$, we note that $M[1]=P[2] \simeq \tau P[1] \simeq I$, so applying $\operatorname{Hom}_{\mathcal{C}}(N$, ) to triangle (2), we obtain the exact sequence

$$
0 \rightarrow \operatorname{Hom}_{\mathcal{C}}\left(N, B^{\prime}\right) \rightarrow \operatorname{Hom}_{\mathcal{C}}\left(N, M^{*}\right) \rightarrow 0,
$$

noting that $\operatorname{Hom}_{\mathcal{C}}(N, M)=\operatorname{Hom}_{\mathcal{C}}(N, P[1])=0$, and (b) follows.

The argument in case $M^{*}$ is not a module is similar.

Now assume that $N$ is a preprojective or preinjective module, or $N$ is a shift of a projective. Then $\tau^{n} N$ is projective for some $n \in \mathbb{Z}$. Since $M \not \tau N$, then $\tau^{n} M \nsucceq \tau\left(\tau^{n} N\right)$, and similarly for $M^{*}$. By the above, either (a) or (b) holds for $\tau^{n}(N)$, so one of

$$
0 \rightarrow \operatorname{Hom}_{\mathcal{C}}\left(\tau^{n} N, \tau^{n} M^{*}\right) \rightarrow \operatorname{Hom}_{\mathcal{C}}\left(\tau^{n} N, \tau^{n} B\right) \rightarrow \operatorname{Hom}_{\mathcal{C}}\left(\tau^{n} N, \tau^{n} M\right) \rightarrow 0,
$$

$\mathrm{Or}$

$$
0 \rightarrow \operatorname{Hom}_{\mathcal{C}}\left(\tau^{n} N, \tau^{n} M\right) \rightarrow \operatorname{Hom}_{\mathcal{C}}\left(\tau^{n} N, \tau^{n} B^{\prime}\right) \rightarrow \operatorname{Hom}_{\mathcal{C}}\left(\tau^{n} N, \tau^{n} M^{*}\right) \rightarrow 0
$$

is exact. Hence one of

$$
0 \rightarrow \operatorname{Hom}_{\mathcal{C}}\left(N, M^{*}\right) \rightarrow \operatorname{Hom}_{\mathcal{C}}(N, B) \rightarrow \operatorname{Hom}_{\mathcal{C}}(N, M) \rightarrow 0,
$$

or

$$
0 \rightarrow \operatorname{Hom}_{\mathcal{C}}(N, M) \rightarrow \operatorname{Hom}_{\mathcal{C}}\left(N, B^{\prime}\right) \rightarrow \operatorname{Hom}_{\mathcal{C}}\left(N, M^{*}\right) \rightarrow 0
$$

is exact (since $\tau$ is an autoequivalence of $\mathcal{C}$ ) and we are done.

Corollary 4.2. Each indecomposable preprojective module, preinjective module, or shift of an indecomposable projective module is exchange compatible.

Proof. This follows from Proposition 4.1 
Proof of Theorem 1.5: We observe that Theorem 1.5(a) follows from Corollary 4.2 and Theorem 1.5 (b) follows from Corollary 2.5.

Proof of Corollary 1.7; If $Q$ is Dynkin, then $k Q$ has no regular modules, and if $Q$ has only two vertices, there are no exceptional regular modules. It follows from Theorem 1.5(a) that in these cases, every cluster variable has a $T$-denominator for every cluster-tilting object $T$. Conversely, suppose that every cluster variable has a $T$-denominator for every cluster-tilting object $T$. We note that any exceptional indecomposable $k Q$-module can be completed to a tilting module and therefore to a cluster-tilting object by BMRRT, 3.3]. If $Q$ was not Dynkin and had more than two vertices, $k Q$ would have an indecomposable regular exceptional module $N$ of maximal quasilength amongst the indecomposable exceptional modules in its ARquiver component (see [Ri2] for the wild case). By Proposition 2.6, we would have $\operatorname{End}_{\mathcal{C}}(N) \not k$, a contradiction to Theorem 1.5 (b).

\section{CRiteria FOR EXACTNESS}

In this section we shall interpret exchange compatibility further. The results here hold for any finite dimensional path algebra. They will be used in the tame case in the next section. To ease notation we $\operatorname{let} \operatorname{Hom}_{\mathcal{D}}()=,($,$) and \operatorname{Ext}_{\mathcal{D}}^{1}()=,{ }^{1}($, (note that these coincide with the corresponding Hom and $\operatorname{Ext}^{1}$-spaces over $H$ if both objects are $H$-modules). We also let $\operatorname{Hom}_{\mathcal{C}_{H}}()=,(,)_{\mathcal{C}}$ and $\operatorname{Ext}_{\mathcal{C}_{H}}^{1}()=$, ${ }^{1}(,)_{\mathcal{C}}$.

Lemma 5.1. Let $0 \rightarrow M^{*} \stackrel{f}{\rightarrow} B \stackrel{g}{\rightarrow} M \rightarrow 0$ be an exact exchange sequence of $H$-modules, and let $N$ be an exceptional indecomposable object in $\mathcal{C}$.

(a) $(N,)_{\mathcal{C}}$ gives a right exact sequence if and only if any $H$-map $h: N \rightarrow M$ factors through $g$.

(b) $(N,)_{\mathcal{C}}$ gives a left exact sequence if and only if any $H-$ map $s: \tau N \rightarrow M$ factors through $g$.

Proof. Applying $(N,)_{\mathcal{C}}$ gives the long exact sequence

$$
\begin{aligned}
& 0 \longrightarrow\left(N, M^{*}\right) \longrightarrow(N, B) \longrightarrow(N, M) \longrightarrow \\
& \text { Ш } \quad \amalg \quad \amalg \\
& 0 \rightarrow\left(\tau N, M^{*}\right) \rightarrow(\tau N, B) \rightarrow(\tau N, M) \rightarrow^{1}\left(\tau N, M^{*}\right) \rightarrow^{1}(\tau N, B) \rightarrow{ }^{1}(\tau N, M) \rightarrow 0
\end{aligned}
$$

The claim follows directly from this.

Remark 5.2. Given a finite number of indecomposable objects in a cluster category, where each of them is preprojective, preinjective or the the shift of a projective, then by changing the hereditary algebra $H$, if necessary (i.e. via a tilt), we can assume that in fact all objects are preprojective (or preinjective). We make use of this in the sequel.

Lemma 5.3. Let $M \rightarrow B^{\prime} \rightarrow M^{*} \rightarrow$ be an exchange triangle, where $M$ is regular and $M^{*}$ is preprojective. Let $N$ be an indecomposable regular exceptional module.

(a) $(N,)_{\mathcal{C}}$ gives a left exact sequence if and only if for any nonzero $F$-map $N \rightarrow M$ the composition $N \rightarrow M \rightarrow B^{\prime}$ is nonzero.

(b) $(N,)_{\mathcal{C}}$ gives a right exact sequence if and only if for any nonzero $F$-map $\tau^{-1} N \rightarrow M$ the composition $\tau^{-1} N \rightarrow M \rightarrow B^{\prime}$ is nonzero.

Proof. (a) One direction is obvious. Assume that for any nonzero $F$-map $N \rightarrow M$ the composition $N \rightarrow M \rightarrow B^{\prime}$ is nonzero, then we need to see that the map 
$(N, M)_{\mathcal{C}} \rightarrow\left(N, B^{\prime}\right)_{\mathcal{C}}$ is injective. Any non-zero map $N \rightarrow M$ in $\mathcal{C}$ must have the form $\left(h, h^{\prime}\right)$, where $h$ is an $H$-map and $h^{\prime}$ is an $F$-map, by [BMRRT, 1.5]. Suppose, for a contradiction, that its composition with the map from $M$ to $B^{\prime}$ is zero. Then there is a map $s: N \rightarrow \tau^{-1} M^{*}$ such that $\left(h, h^{\prime}\right)=t s$, where $t: \tau^{-1} M^{*} \rightarrow M$. Since $\tau^{-1} M^{*}$ is preprojective and $M$ is regular, we have that $\left(\tau^{-1} M^{*}, F M\right)=$ $\left(\tau^{-1} M^{*}, \tau^{-1} M[1]\right) \simeq\left(M^{*}, M[1]\right) \simeq{ }^{1}\left(M^{*}, M\right) \simeq D\left(M, \tau M^{*}\right)=0$ since $\tau M^{*}$ is preprojective. It follows that $t$ is an $H$-map. Similarly, $\left(N, \tau^{-1} M^{*}\right)=0$ since $N$ is regular and $\tau^{-1} M^{*}$ is preprojective, so $s$ is an $F$-map. Comparing $H$-maps and $F$-maps, we see that $h=0$ and $h^{\prime}=t s$. Hence the $F$-map $h^{\prime}$ is non-zero while its composition with the map from $M$ to $B^{\prime}$ is zero, contradicting the assumption in (a).

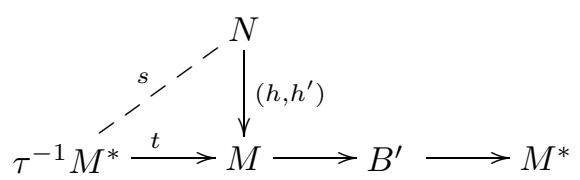

(b) There is a long exact sequence

$$
(N, M)_{\mathcal{C}} \rightarrow\left(N, B^{\prime}\right)_{\mathcal{C}} \rightarrow\left(N, M^{*}\right)_{\mathcal{C}} \rightarrow(N, \tau M)_{\mathcal{C}} \rightarrow\left(N, \tau B^{\prime}\right)_{\mathcal{C}} \rightarrow\left(N, \tau M^{*}\right)_{\mathcal{C}}
$$

Hence $\left(N, B^{\prime}\right)_{\mathcal{C}} \rightarrow\left(N, M^{*}\right)_{\mathcal{C}}$ is an epimorphism if and only if $(N, \tau M)_{\mathcal{C}} \rightarrow\left(N, \tau B^{\prime}\right)_{\mathcal{C}}$ is a monomorphism. This clearly holds if and only if $\left(\tau^{-1} N, M\right)_{\mathcal{C}} \rightarrow\left(\tau^{-1} N, B^{\prime}\right)_{\mathcal{C}}$ is a monomorphism. By (a), this holds if and only if for any non-zero $F$-map $\tau^{-1} N \rightarrow M$, the composition $\tau^{-1} N \rightarrow M \rightarrow B^{\prime}$ is nonzero.

We have the following direct consequence of Lemmas 5.1 and 5.3 .

Proposition 5.4. Let $\left(M, M^{*}\right)$ be an exchange pair with $M$ regular and $M^{*}$ preprojective. Let $0 \rightarrow M^{*} \rightarrow B \rightarrow M \rightarrow 0$ and $M \rightarrow B^{\prime} \rightarrow M^{*} \rightarrow$ be the corresponding exchange sequence and triangle, where $B=B_{1} \amalg M_{1}$ and $B^{\prime}=B_{1}^{\prime} \amalg M_{1}^{\prime}$ with $B_{1}, B_{1}^{\prime}$ preprojective and $M_{1}, M_{1}^{\prime}$ regular. Let $N$ be an indecomposable regular exceptional module.

(a) Applying $(N,)_{\mathcal{C}}$ to $0 \rightarrow M^{*} \rightarrow B_{1} \amalg M_{1} \rightarrow M \rightarrow 0$ gives a short exact sequence if and only if any $H-$ map $N \amalg \tau N \rightarrow M$ factors through $M_{1} \rightarrow M$.

(b) Applying $(N,)_{\mathcal{C}}$ to $M \rightarrow B_{1}^{\prime} \amalg M_{1}^{\prime} \rightarrow M^{*} \rightarrow$ gives a short exact sequence if and only if for any nonzero $F$-map $N \amalg \tau^{-1} N \rightarrow M$ the composition $N \amalg \tau^{-1} N \rightarrow M \rightarrow M_{1}^{\prime}$ is nonzero.

Proof. (a) If $N \amalg \tau N \rightarrow M$ is an $H$-map, it cannot factor through $B_{1} \rightarrow M$. We apply Lemma 5.1 .

(b) Since $B_{1}^{\prime}$ is preprojective, the map $M \rightarrow B_{1}^{\prime}$ is an $F$-map. Hence if $N \amalg \tau^{-1} N \rightarrow$ $M$ is an $F$-map, then the composition $N \amalg \tau^{-1} N \rightarrow M \rightarrow B_{1}^{\prime}$, must be zero. We apply Lemma 5.3 .

\section{THE TAME CASE}

In this section, we investigate the case in which $H$ is tame in more detail. In particular, we determine which exceptional regular modules are exchange compatible in this case.

Lemma 6.1. Let $H$ be a tame hereditary algebra and let $\left(M, M^{*}\right)$ be an exchange pair with $M$ regular and $M^{*}$ preprojective. Let $0 \rightarrow M^{*} \rightarrow B \rightarrow M \rightarrow 0$ and $M \rightarrow B^{\prime} \rightarrow M^{*} \rightarrow$ be the corresponding exchange sequence and triangle, where $B=B_{1} \amalg M_{1}$ and $B^{\prime}=B_{1}^{\prime} \amalg M_{1}^{\prime}$ with $B_{1}, B_{1}^{\prime}$ preprojective and $M_{1}, M_{1}^{\prime}$ regular. Then the maps $M_{1} \rightarrow M$ and $M \rightarrow M_{1}^{\prime}$ are $H$-maps. 


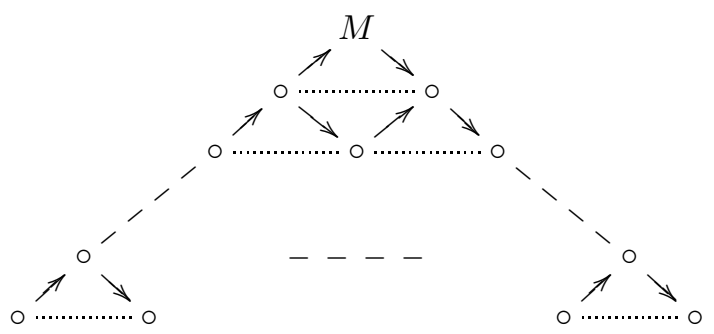

FiguRE 2. The AR-quiver of the subcategory of subfactors of $M$

Proof. The first claim holds obviously, since the exchange sequence is in mod $H$. For the second claim, by Remark 5.2 , by replacing $H$ with $H^{\prime}$ via a tilt, we can assume that $M^{*}$ is preinjective, and similarly that $B_{1}^{\prime}$ is preinjective. Then the triangle $M \rightarrow B^{\prime} \rightarrow M^{*} \rightarrow$ is the image of an exact sequence of $H^{\prime}$-modules $0 \rightarrow H^{\prime} M \rightarrow H^{\prime} B^{\prime} \rightarrow H^{\prime} M^{*} \rightarrow 0$. Then $M \rightarrow M_{1}^{\prime}$ is an $H^{\prime}$-map and consequently also an $H$-map.

Remark 6.2. Let $\mathcal{E}$ denote a category which is either the module category of the path algebra $\Lambda=\Lambda_{n}$ of a quiver of type $A_{n}$ with linear orientation, or the abelian category associated with a tube, i.e. a connected component of regular modules over some tame hereditary algebra $\Lambda$. For an indecomposable exceptional object $M$ in $\mathcal{E}$, we let $\Delta_{M}$ denote the additive subcategory generated by the subfactors of $M$ inside $\mathcal{E}$, or equivalently the extension closure of $\operatorname{Sub} M \cup$ Fac $M$. The indecomposable objects in $\Delta_{M}$ form a full subquiver of the AR-quiver of $\Lambda$, which is a triangle with $M$ sitting on the top (see Figure 2).

Lemma 6.3. Consider the quiver of type $A_{t}$ with linear orientation and let $\Lambda$ be its path algebra. Let $B$ be the indecomposable projective and injective $\Lambda$-module. Assume $B_{1} \rightarrow B_{2} \rightarrow \cdots \rightarrow B_{s} \rightarrow B \rightarrow C_{1} \rightarrow \cdots \rightarrow C_{t-s}$ is a sequence of irreducible maps, where $t>s>0$. If $T$ is a tilting module over $\Lambda$, it must contain one of the $B_{i}($ for $1 \leq i \leq s)$ or $C_{j}$ (for $1 \leq j \leq t-s$ ) as an indecomposable direct summand.

Proof. Assume to the contrary that $T$ is a tilting module having none of the $B_{i}, C_{j}$ as indecomposable direct summands. The object $B$ is necessarily a summand of $T$, since it is projective-injective. Since $B_{s}$ only has extensions with the the proper factors of $B$, at least one such factor must be a summand of $T$. Let $E_{j} \in$ Fac $B \cap$ $\operatorname{add}(T / B)$, with length $j$ maximal. Then, by assumption $j \leq t-(t-s)-1=s-1$.

Let $P_{i}$ be the indecomposable projective of length $i$, and let $k=t-j-1$. Then $T / B$ is in $\Delta_{E_{j}} \cup \Delta_{P_{k}}$, since, for $k<l<t, P_{l}$ and all factors of $P_{l}$ which are not in $\Delta_{E_{j}}$ have non-trivial extensions with $E_{j}$. Note that $T / B$ has $t-1$ nonisomorphic indecomposable summands. The subcategory $\Delta_{E_{j}}$ has clearly at most $j$ summands in $T$, so at least $t-1-j=k$ summands $T_{1}, \ldots, T_{t-(j+1)}$ of $T$ are in $\Delta_{P_{k}}$. Note that $\Delta_{P_{k}}$ is clearly equivalent to $\bmod \Lambda_{k}$, so $T^{\prime}=T_{1} \amalg \cdots \amalg T_{t-(j+1)}$ forms a tilting module in this subcategory, and hence $P_{k}$ is a summand in $T^{\prime}$. But this is a contradiction, since $P_{k}$ is equal to some $B_{i}$. We illustrate the situation in Figure 3 ,

Remark 6.4. Let $M$ be an indecomposable regular module in a tube $\mathcal{T}$ of rank $n$ for a tame hereditary algebra $H$. Assume q. l. $M=t \leq n-1=\operatorname{rank} \mathcal{T}$ (where q. l. denotes quasilength). Let $\Lambda_{t}$ be the path algebra of a quiver of type $A_{t}$ with linear orientation. Then there is a functor $F_{t}: \bmod \Lambda_{t} \rightarrow \Delta_{M} \subseteq \mathcal{T}$, which is 


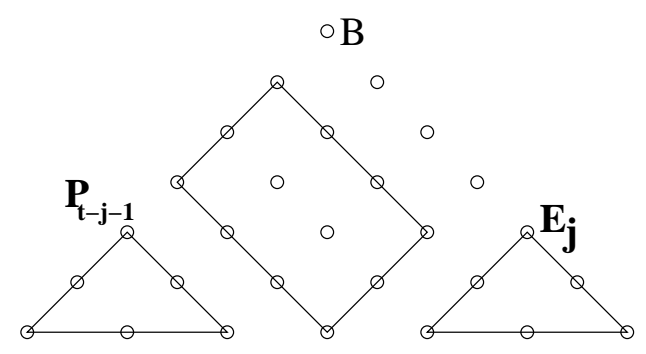

Figure 3. Proof of Lemma 6.3.

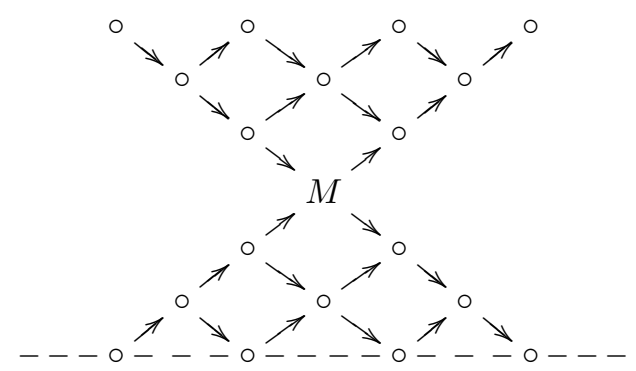

Figure 4. Proof of Lemma 6.5 The dotted line indicates the $\tau$-orbit of quasi-simple objects in the tube.

clearly fully faithful (from the structure of the tube), exact and dense and preserves indecomposability.

The following is due to Strauss [ $\underline{\mathrm{S}}$, Cor. 3.7].

Lemma 6.5. Let $M$ be an indecomposable exceptional $H$-module for $H$ tame hereditary, lying in a tube $\mathcal{T}$ of rank $n$. Let $\mathrm{q} \cdot \mathrm{l} .(M)=t$ (note that necessarily $t \leq n-1$ ). Let $T=T^{\prime} \amalg M$ be a tilting $H$-module. Then $\operatorname{add} T \cap \Delta_{M}=\operatorname{add} \widetilde{T_{0}}$, where $\widetilde{T_{0}}=F_{t}\left(T_{0}\right)$, for a tilting module $T_{0}$ in $\bmod \Lambda_{t}$.

Lemma 6.6. Let $\mathcal{T}$ be a tube of rank $n \geq 3$. Let $N, M$ be indecomposable objects in $\mathcal{T}$ with q. l. $(N)=j$, where $1 \leq j \leq n-2$, and q. l. $(M)=t$, where $1 \leq t \leq n-1$. Suppose that

$$
X_{1} \rightarrow X_{2} \rightarrow \cdots \rightarrow X_{t}=M
$$

is a sequence of irreducible maps with $X_{t}=M$ and q.1. $\left(X_{i}\right)=i$ and that

$$
M=Y_{t} \rightarrow Y_{t-1} \rightarrow \cdots \rightarrow Y_{1}
$$

is a sequence of irreducible maps with $Y_{t}=M$ and q. l. $\left(Y_{i}\right)=i$.

(a) Let $f \in \operatorname{Hom}_{\mathcal{T}}(N, M)$ and $g \in \operatorname{Hom}_{\mathcal{T}}\left(M, \tau^{2} N\right)$ be non-zero maps. Then the number of modules $X_{i}, 1 \leq i<t$, that $f$ factors through plus the number of modules $Y_{i}, 1 \leq i<t$, that $g$ factors through is at least $t$.

(b) Statement (a) holds if we assume instead that $f \in \operatorname{Hom}_{\mathcal{T}}(\tau N, M)$ or $g \in$ $\operatorname{Hom}_{\mathcal{T}}(M, \tau N)$ or both.

Proof. (a) Suppose first that $j \geq t$. Let

$$
N=U_{j} \rightarrow U_{j-1} \rightarrow \cdots \rightarrow U_{1}
$$

be a sequence of irreducible maps with $N=U_{j}$ and q.l. $\left(U_{i}\right)=i$ for all $i$. Then $U_{t}=\tau^{a}(M)$ for some $a$, where $1 \leq a \leq n-1$, so $U_{t-a}=X_{t-a}$. From the structure 


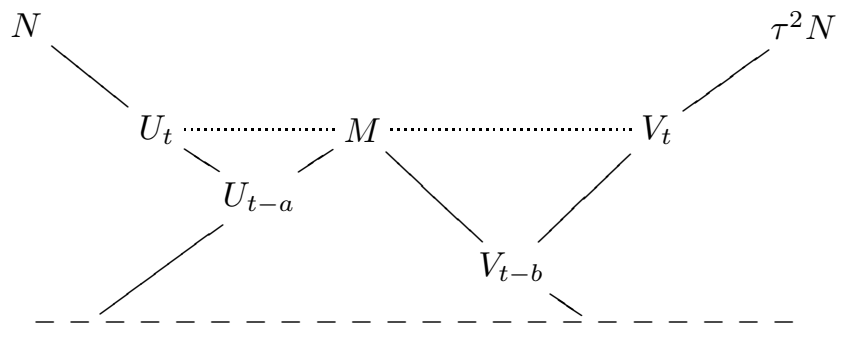

Figure 5. Proof of Lemma 6.6 (the dotted line indicates the $\tau$ orbit of quasi-simple objects in the tube).

of the tube it follows that $f$ factors through $X_{t-a}, X_{t-a+1}, \ldots, X_{t-1}$, a total of $a$ modules.

Let

$$
V_{1} \rightarrow V_{2} \rightarrow \cdots \rightarrow V_{j}=\tau^{2} N
$$

be a sequence of irreducible maps with $V_{j}=\tau^{2} N$ and q. l. $\left(V_{i}\right)=i$ for all $i$. Then $V_{t}=\tau^{-b}(M)$ for some $b$, where $1 \leq b \leq n-1$, so $V_{t-b}=Y_{t-b}$. From the structure of the tube it follows that $g$ factors through $Y_{t-b}, Y_{t-b+1}, \ldots, Y_{t-1}$, a total of $b$ modules.

Since $\tau^{n-2}\left(\tau^{2}(N)\right)=N$ and q. l. $(N)=j$, we have that $\tau^{n-2-(j-t)}\left(V_{t}\right)=U_{t}$, so $a+b=n-2-(j-t)=(n-2-j)+t \geq t$ since we have assumed $j \leq n-2$. See Figure 5 .

The argument in case $j<t$ is similar, so we are done.

For (b) we just need to remark that a non-zero map $\tau N \rightarrow M$ factors through more of the $X_{i}$ than $f$ does, and that a non-zero map $M \rightarrow \tau N$ factors through more of the $Y_{i}$ than $g$ does. Therefore (a) holds if $N$ is replaced by $\tau N$, or $\tau^{2} N$ is replaced by $\tau N$, or both. Statement (b) follows.

Lemma 6.7. Let $H$ be tame and $N$ indecomposable exceptional in a tube of rank $n \geq 2$, so that q. l. $(N) \leq n-1$. Then q. l. $(N)=n-1$ if and only if $\operatorname{dim}_{k} \operatorname{End}_{\mathcal{C}}(N)>$ 1. In this case, there is a non-zero $F$-map from $N$ to $N$ and $\operatorname{dim}_{k} \operatorname{End}_{\mathcal{C}}(N)=2$.

Proof. It is easy to see that $\operatorname{Hom}_{H}\left(N, \tau^{2} N\right) \neq 0$ (and is one-dimensional) if and only if q. l. $(N)=n-1$, using $\tau^{2} N=\tau^{-(n-2)} N$. Furthermore, $\operatorname{Hom}_{\mathcal{C}}(N, N)=$ $\operatorname{Hom}(N, N) \amalg \operatorname{Hom}(N, F N)$. We have that $\operatorname{Hom}(N, F N) \simeq \operatorname{Ext}^{1}\left(N, \tau^{-1} N\right)$, and $\operatorname{Ext}^{1}\left(N, \tau^{-1} N\right) \simeq D \operatorname{Hom}\left(N, \tau^{2} N\right)$.

Lemma 6.8. Let $H$ be tame and $N$ indecomposable exceptional in a tube of rank $n \geq 2$, with q.1. $(N)=j \leq n-2$. Let $\left(M, M^{*}\right)$ be an exchange pair, where $M$ and $M^{*}$ are complements of some almost complete cluster-tilting object $\bar{T}^{\prime}$. We assume that $M$ is regular. Then we have an exchange sequence $0 \rightarrow M^{*} \rightarrow B \rightarrow M \rightarrow 0$ and exchange triangle $M \rightarrow B^{\prime} \rightarrow M^{*} \rightarrow$. Write $B=B_{1} \amalg M_{1}$ and $B^{\prime}=B_{1}^{\prime} \amalg M_{1}^{\prime}$, where $M_{1}, M_{1}^{\prime}$ are regular and $B_{1}, B_{1}^{\prime}$ are preprojective. Then either:

(i) any $H$-map $N \amalg \tau N \rightarrow M$ factors through $B \rightarrow M$, or

(ii) for any nonzero $F$-map $N \amalg \tau^{-1} N \rightarrow M$, the composition $N \amalg \tau^{-1} N \rightarrow$ $M \rightarrow M_{1}^{\prime}$ is nonzero.

Proof. We can clearly assume that there is some nonzero $H$-map $N \amalg \tau N \rightarrow M$ and some nonzero $F$-map $N \amalg \tau^{-1} N \rightarrow M$, and hence there is some nonzero $H$-map $M \rightarrow \tau^{2} N \amalg \tau N$. In particular, $N$ and $M$ are in the same tube. By Lemma 6.5, 
$\bar{T}^{\prime}$ is the direct sum of the image under $F_{t}$ of a tilting module $T_{0}$ in $\bmod \Lambda_{t}$ and another summand (using the notation of Remark 6.4). Let $\widetilde{T_{0}}=F_{t}\left(T_{0}\right)$.

Case (I):

We first assume that there is a non-zero $H$-map from $N$ to $M$ and a non-zero $H$ map from $M$ to $\tau^{2} N$. By Lemma 6.3, combined with Lemma 6.6(a), we see that either some non-zero $H$-map from $N$ to $M$ factors through some indecomposable direct summand of $\bar{T}^{\prime}$ or that some non-zero $H$-map $M \rightarrow \tau^{2} N$ factors through some indecomposable direct summand of $\bar{T}^{\prime}$.

In the first case, we note that, since $\operatorname{Hom}_{H}(N, M) \simeq k$, any non-zero $H$-map from $N$ to $M$ factors through $\bar{T}^{\prime}$. Since any non-zero $H$-map from $\tau N$ to $M$ must factor through an $H$-map from $N$ to $M$, it follows that any non-zero $H$-map from $N \amalg \tau N$ to $M$ must factor through $\bar{T}^{\prime}$, and therefore through $B \rightarrow M$, and we see that (i) holds.

In the second case, arguing similarly, we see that any non-zero $H$-map from $M$ to $\tau^{2} N \amalg \tau N$ factors through the $H$-map component of $M \rightarrow B^{\prime}$ (noting that an $H$-map between two modules cannot factor through an $F$-map). It follows that the map from $\operatorname{Hom}\left(B^{\prime}, \tau^{2} N \amalg \tau N\right)$ to $\operatorname{Hom}\left(M, \tau^{2} N \amalg \tau N\right)$ given by composition with the $H$-map component of $M \rightarrow B^{\prime}$ is surjective, so that the induced map from $\left.D \operatorname{Hom}\left(M, \tau^{2} N \amalg \tau N\right)\right)$ to $\left.D \operatorname{Hom}\left(B^{\prime}, \tau^{2} N \amalg \tau N\right)\right)$ is injective. Hence the induced map from $\left.D \operatorname{Hom}\left(\tau^{-1} M, \tau N \amalg N\right)\right)$ to $\left.D \operatorname{Hom}\left(\tau^{-1} B^{\prime}, \tau N \amalg N\right)\right)$ is injective. So the map from $\operatorname{Ext}^{1}\left(N \amalg \tau^{-1} N, \tau^{-1} M\right)$ to $\operatorname{Ext}^{1}\left(N \amalg \tau^{-1} N, \tau^{-1} B^{\prime}\right)$ is injective, and therefore the map from $\operatorname{Hom}\left(N \amalg \tau^{-1} N, \tau^{-1} M[1]\right)$ to $\operatorname{Hom}\left(N \amalg \tau^{-1} N, \tau^{-1} B^{\prime}[1]\right)$ is injective. Hence the map from $\operatorname{Hom}\left(N \amalg \tau^{-1} N, F M\right)$ to $\operatorname{Hom}\left(N \amalg \tau^{-1} N, F B^{\prime}\right)$ is injective, and we see that the composition of any non-zero $F$-map from $N \amalg \tau^{-1} N$ to $M$ with the $H$-map component of the map $M \rightarrow B^{\prime}$ is non-zero. Note that composition of any such $F$-map with the $F$-map component is always zero. It follows that the composition of any non-zero $F$-map from $N \amalg \tau^{-1} N$ to $M$ with the map $M \rightarrow B^{\prime}$ is non-zero. We see that (ii) holds and we are done.

Case (II):

We now assume that $\operatorname{Hom}_{H}(N, M)=0$, that there is a non-zero $H$-map from $\tau N$ to $M$ and a non-zero $H$-map from $M$ to $\tau^{2} N$. By Lemma 6.3 combined with Lemma 6.6(b), we see that either some non-zero $H$-map from $\tau N$ to $M$ factors through some summand of $\bar{T}^{\prime}$ or that some non-zero $H$-map $M \rightarrow \tau^{2} N$ factors through some summand of $\bar{T}^{\prime}$.

In the first case, we note that, since $\operatorname{Hom}(\tau N, M) \simeq k$, any non-zero $H$-map from $\tau N$ to $M$ factors through $\bar{T}^{\prime}$. Since $\operatorname{Hom}_{H}(N, M)=0$, we see that any non-zero $H$-map from $N \amalg \tau N$ to $M$ must factor through $\bar{T}^{\prime}$, and therefore through $B \rightarrow M$, and we see that (i) holds.

The argument in the second case is as in Case (I).

Case (III):

We now assume that there is a non-zero $H$-map from $N$ to $M$, that $\operatorname{Hom}_{H}\left(M, \tau^{2} N\right)=$ 0 and that there is a non-zero $H$-map from $M$ to $\tau N$. By Lemma 6.3. combined with Lemma 6.6(b), we see that either some non-zero $H$-map from $N$ to $M$ factors through some summand of $\bar{T}^{\prime}$ or that some non-zero $H$-map $M \rightarrow \tau N$ factors through some summand of $\bar{T}^{\prime}$.

The argument in the first case is as in Case (I).

In the second case, since $\operatorname{Hom}_{H}\left(M, \tau^{2} N\right)=0$, we see that any non-zero $H$-map from $M$ to $\tau^{2} N \amalg \tau N$ factors through the $H$-map component of $M \rightarrow B^{\prime}$ (noting that an $H$-map between two modules cannot factor through an $F$-map). As in Case (I), we see that (ii) holds. 
Case (IV):

Finally, we assume that $\operatorname{Hom}_{H}(N, M)=0, \operatorname{Hom}_{H}\left(M, \tau^{2} N\right)=0$, that there is a non-zero $H$-map from $\tau N$ to $M$ and that there is a non-zero $H$-map from $M$ to $\tau N$. By Lemma 6.3, combined with Lemma 6.6(b), we see that either some non-zero $H$ map from $\tau N$ to $M$ factors through some summand of $\bar{T}^{\prime}$ or that some non-zero $H$-map $M \rightarrow \tau N$ factors through some summand of $\bar{T}^{\prime}$.

In the first case we argue as in Case (II), and in the second case we argue as in Case (III), and we are done.

Theorem 6.9. Let $H$ be tame and $N$ indecomposable exceptional in a tube of rank $n \geq 2$, with q.l. $(N)=j \leq n-2$. Then $N$ is exchange compatible.

Proof. Let $\left(M, M^{*}\right)$ be an exchange pair, with $M$ and $M^{*}$ complements of some almost complete cluster-tilting object $\bar{T}^{\prime}$.

Case (I):

We assume first that $M$ is regular and $M^{*}$ preprojective. Then we have an exchange sequence $0 \rightarrow M^{*} \rightarrow B \rightarrow M \rightarrow 0$ and exchange triangle $M \rightarrow B^{\prime} \rightarrow M^{*} \rightarrow$. Write $B=B_{1} \amalg M_{1}$ and $B^{\prime}=B_{1}^{\prime} \amalg M_{1}^{\prime}$, where $M_{1}, M_{1}^{\prime}$ are regular and $B_{1}, B_{1}^{\prime}$ are preprojective. By Lemma 6.8, either

(i) any $H$-map $N \amalg \tau N \rightarrow M$ factors through $B \rightarrow M$, or

(ii) for any nonzero $F$-map $N \amalg \tau^{-1} N \rightarrow M$, the composition $N \amalg \tau^{-1} N \rightarrow$ $M \rightarrow M_{1}^{\prime}$ is nonzero.

If (i) holds, we see by Lemma 5.1 that the sequence (3) is exact. If (ii) holds, we see by Lemma 5.3 that the sequence (4) is exact.

Case (II):

Next, we assume that $M$ and $M^{*}$ are both preprojective. We can still assume that we have an exchange sequence $0 \rightarrow M^{*} \rightarrow B \rightarrow M \rightarrow 0$ of $H$-modules, so that $B$ is also preprojective. Applying $(N,)_{\mathcal{C}}$, arguing as in Lemma 5.1 and using that $\operatorname{Hom}_{H}(N, X)=0$ for any preprojective $X$ we see that the long exact sequence

$$
\left(N, \tau^{-1} M\right)_{\mathcal{C}} \rightarrow\left(N, M^{*}\right)_{\mathcal{C}} \rightarrow(N, B)_{\mathcal{C}} \rightarrow(N, M)_{\mathcal{C}} \rightarrow\left(N, \tau M^{*}\right)_{\mathcal{C}} \rightarrow
$$

reduces to

$$
0 \rightarrow \operatorname{Ext}_{H}^{1}\left(N, \tau^{-1} M^{*}\right) \rightarrow \operatorname{Ext}_{H}^{1}\left(N, \tau^{-1} B\right) \rightarrow \operatorname{Ext}_{H}^{1}\left(N, \tau^{-1} M\right) \rightarrow 0 .
$$

Case (III):

Finally, we consider the case where both $M$ and $M^{*}$ are regular modules. By Lemma 5.1 (and arguing as in Lemma 5.3) we see that it is enough to show that either

(a) Any $H$-map $N \amalg \tau N \rightarrow M$ factors through $g$ in the sequence

$$
0 \rightarrow M^{*} \stackrel{f}{\rightarrow} B \stackrel{g}{\rightarrow} M \rightarrow 0
$$

or

(b) For any non-zero map $N \amalg \tau^{-1} N \rightarrow M$, the composition with $h$ is non-zero, where $h$ is the map in the triangle:

$$
\tau^{-1} M^{*} \rightarrow M \stackrel{h}{\rightarrow} B^{\prime} \stackrel{k}{\rightarrow} M^{*} .
$$

Note that statement (a) is the same as statement 6.8(i). In order to do this, it is clear that we can assume that there is a non-zero $H$-map from $N \amalg \tau N$ to $M$ and that there is a non-zero map (in $\mathcal{C}$ ) from $N \amalg \tau^{-1} N$ to $M$. From the former, it follows that $N$ and $M$ must lie in the same tube.

We first of all show the following claim:

Claim: If there is a non-zero H-map from $N \amalg \tau^{-1} N$ to $M$ whose composition with $h$ is zero then any non-zero $H$-map from $N \amalg \tau N$ to $M$ factors through $g: B \rightarrow M$. 
Proof of claim: If $\gamma: N \rightarrow M$ is a non-zero $H$-map and the composition $h \circ \gamma$ is zero, then $\gamma$ lifts to $\tau^{-1} M^{*}$. It follows that $\tau^{-1} M^{*}$ lies in the rectangle spanned by $N$ and $M$ in the tube (see Figure 6). Hence $M^{*}$ lies in the rectangle spanned by $\tau M$ and $\tau N$ in the tube (the dotted rectangle in Figure 6). The middle term in the short exact sequence (17) is the direct sum of two indecomposable modules, $B_{1}$ and $B_{2}$, which are at the other two corners of the rectangle spanned by $M$ and $M^{*}$. We see from the structure of the tube that $\gamma$ factors through at least one of $B_{1}$ and $B_{2}$ (using the fact that $M^{*} \nsucceq \tau N$ ). It follows that $\gamma$ factors through the map $B \stackrel{g}{\rightarrow} M$. Since $\operatorname{Hom}_{H}(N, M) \simeq k$, we see that every $H$-map from $N$ to $M$ factors through $B \stackrel{g}{\rightarrow} M$.

Since a non-zero $H$-map from $\tau N$ to $M$ factors through any $H$-map from $N$ to $M$ (note that such maps, if they exist, are unique up to a scalar multiple), it follows that any non-zero $H$-map from $\tau N$ to $M$ factors through $B \stackrel{g}{\rightarrow} M$ also. Thus any non-zero $H$-map from $N \amalg \tau N$ to $M$ factors through $B \stackrel{g}{\rightarrow} M$.

If $\gamma: \tau^{-1} N \rightarrow M$ is a non-zero $H$-map and its composition with $h$ is zero, then, as above, $\gamma$ must factor through $B \stackrel{g}{\rightarrow} M$. Then any non-zero $H$-map from $N$ to $M$ must factor through $\gamma$ and therefore, by the above, through $B \stackrel{g}{\rightarrow} M$. Again arguing as above, we see that any non-zero $H$-map from $\tau N$ to $M$ factors through $B \stackrel{g}{\rightarrow} M$ and therefore that any non-zero $H$-map from $N \amalg \tau N$ to $M$ factors through $B \stackrel{g}{\rightarrow} M$. The claim now follows.

Since $M$ is regular, Lemma 6.8 applies, and we see that either 6.8(i) or (ii) holds. If (i) holds, we are done, as (a) above is the same as 6.8(i). If 6.8(ii) holds, it follows that for any non-zero $F$-map $N \amalg \tau^{-1} N \rightarrow M$, the composition $N \amalg \tau^{-1} N \rightarrow M \rightarrow B^{\prime}$ is nonzero.

By the claim above, we know that if there is a non-zero $H$-map $N \amalg \tau^{-1} N \rightarrow M$ whose composition with $h$ is zero then (a) holds, and we are done. So we are left with the case in which for any non-zero $H$-map or $F$-map from $N \amalg \tau^{-1} N$ to $M$ the composition with $h$ is non-zero.

We claim that this means that for any non-zero map from $N \amalg \tau^{-1} N$ to $M$, the composition $N \amalg \tau^{-1} N \rightarrow M \rightarrow B^{\prime}$ is non-zero. We write maps in $\mathcal{C}$ between indecomposable objects as pairs $\left(f, f^{\prime}\right)$ where $f$ is an $H$-map and $f^{\prime}$ is an $F$-map. Let $\left(h, h^{\prime}\right): N \rightarrow M$ be a non-zero map such that the composition with $M \rightarrow B^{\prime}$ is zero. Then there are maps $\left(t, t^{\prime}\right): N \rightarrow \tau^{-1} M^{*}$ and $\left(s, s^{\prime}\right): \tau^{-1} M^{*} \rightarrow M$ such that $\left(h, h^{\prime}\right)=\left(s, s^{\prime}\right) \circ\left(t, t^{\prime}\right)$. Then $h=s t$, so that $h: N \rightarrow M$ composed with $M \rightarrow B^{\prime}$ is zero; hence $h=0$ (as $h$ is a $H$-map). Then $h^{\prime}$ composed with $M \rightarrow B^{\prime}$ is zero, so $h^{\prime}=0$ (as $h^{\prime}$ is an $F$-map). It follows that $\left(h, h^{\prime}\right)=0$, a contradiction. See Figure 7

We see that the map from $\left(N \amalg \tau^{-1} N, M\right)_{\mathcal{C}}$ to $\left(N \amalg \tau^{-1} N, B^{\prime}\right)_{\mathcal{C}}$ is injective, and thus that (b) above holds.

We can now conclude that if $M \nsucceq \tau N \not M^{*}$, then

$$
\begin{aligned}
\operatorname{dim} \operatorname{Hom}_{\mathcal{C}}(N, M)+\operatorname{dim} \operatorname{Hom}_{\mathcal{C}}\left(N, M^{*}\right) & \\
& =\max \left(\operatorname{dim} \operatorname{Hom}_{\mathcal{C}}(N, B), \operatorname{dim} \operatorname{Hom}_{\mathcal{C}}\left(N, B^{\prime}\right)\right) .
\end{aligned}
$$

Thus $N$ is exchange compatible, and we are done.

Corollary 6.10. Let $H$ be a tame hereditary algebra, and let $N$ be an indecomposable exceptional object in $\mathcal{C}$. Then $N$ is exchange compatible if and only if $\operatorname{End}_{\mathcal{C}}(N) \simeq k$.

Proof. This follows immediately from Theorem 6.9 and Corollary 2.8 together with Proposition 2.6. 


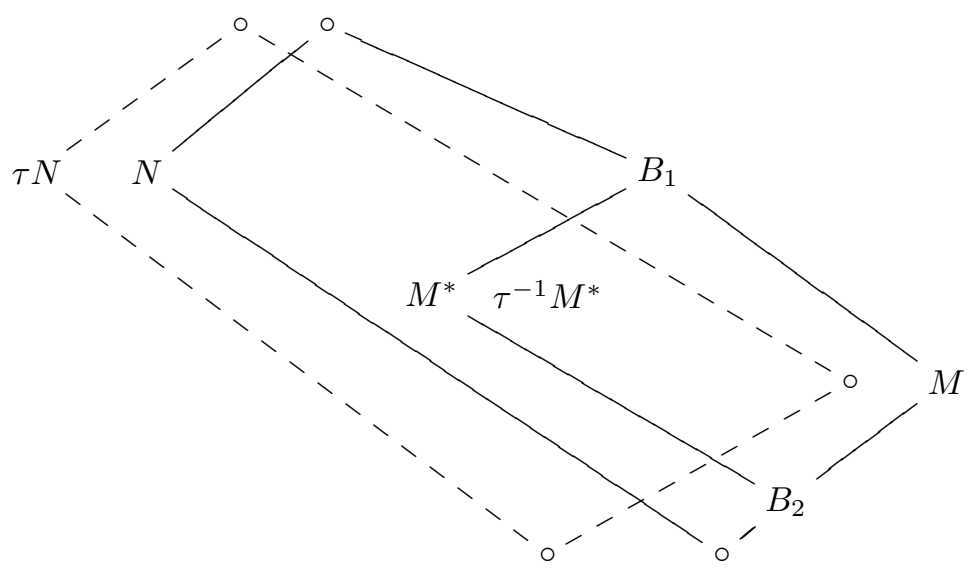

Figure 6. Proof of claim in Theorem 6.9

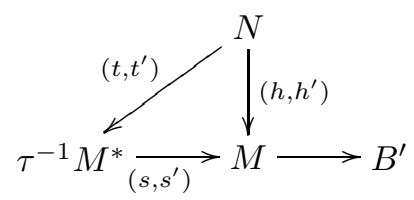

FIGURE 7. End of proof of Theorem 6.9

Proof of Theorem 1.6. By Theorem 3.2, Theorem 1.6(a) is equivalent to the statement:

(a') All indecomposable direct summands $T_{i}$ of $T$ are exchange compatible.

By Lemma 6.7, 1.6(c) implies 1.6(b); by Theorem 6.9. 1.6(b) implies (a'), and by Corollary 2.5. (a') implies 1.6(c). The proof is complete. $\square$.

\section{EXAMPLES}

In this final section we give two examples to illustrate our main result.

Example 7.1. We consider a Dynkin quiver $Q$ of type $A_{3}$, and choose a cluster $\left\{y_{1}, y_{2}, y_{3}\right\}$ with corresponding cyclic quiver $\Gamma$ :

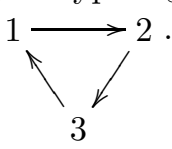

If we choose a cluster-tilting object $T$ where the quiver of $\operatorname{End}_{\mathcal{C}}(T)^{\mathrm{op}}$ is $Q^{\prime}$ and compute all cluster variables with respect to the initial cluster $\left\{y_{1}, y_{2}, y_{3}\right\}$, we get the picture shown in Figure 8, where in the AR-quiver we have represented the indecomposable objects by their corresponding cluster variables.

It is easily verified here that the denominators are all of the prescribed form, as promised by Theorem 1.5 .

Example 7.2. In the next example we consider the quiver $Q$ shown in Figure 9(a). This is mutation equivalent to the quiver $\Gamma$ shown in Figure 9(b). Then the ARquiver of $k Q$ has a tube of rank two with quasisimple modules given by the simple module $S_{2}$ and $M=\tau S_{2}$, a module with composition factors $S_{1}$ and $S_{3}$. We choose a cluster-tilting object $T$ such that the quiver of the cluster-tilted algebra 


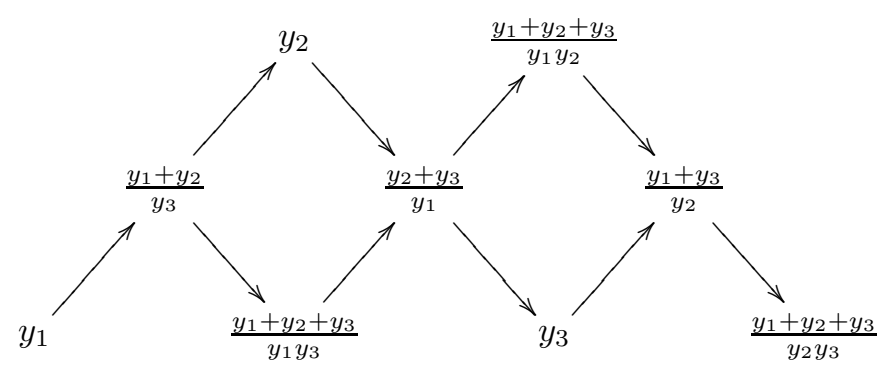

Figure 8. Cluster variables in Example 7.1

(a)

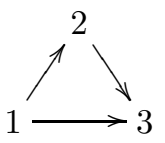

(b)

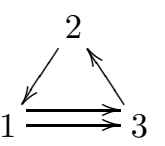

Figure 9. The quivers $Q$ and $\Gamma$ in Example 7.2 .

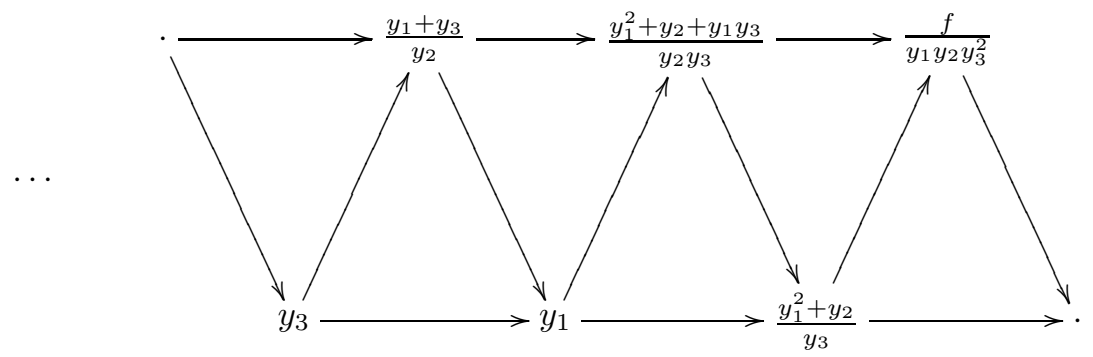

Figure 10. Some cluster variables in type $\widetilde{A_{2}}$

$\operatorname{End}_{C}(T)^{\text {op }}$ is $\Gamma$, for example $T=T_{1} \amalg T_{2} \amalg T_{3}=P_{1} \amalg M \amalg P_{3}$. Then $\tau T=$ $\tau P_{1} \amalg S_{2} \amalg \tau P_{3}$.

Let $y_{i}$ be the cluster variable corresponding to $\tau T_{i}$ for $i=1,2,3$. Then there is a seed $\left(\left\{y_{1}, y_{2}, y_{3}\right\}, \Gamma\right)$ of $\mathcal{A}(Q)$. The cluster variables corresponding to some of the objects in the preprojective/preinjective component of the AR-quiver of $\mathcal{C}_{k Q}$ are shown in Figure 10.

The cluster variables corresponding to the two quasisimple modules in the tube of rank two are $y_{2}$, corresponding to $\tau M=S_{2}$, and $\frac{\left(y_{1}+y_{3}\right)^{2}+y_{2}}{y_{1} y_{2} y_{3}}$ corresponding to $M$. However $\operatorname{Hom}_{\mathcal{C}}\left(T_{2}, M\right)=\operatorname{End}_{\mathcal{C}}(M)$ is easily seen to be two dimensional, while $y_{2}$ appears with multiplicity one in the denominator of the cluster variable corresponding to $M$.

We remark that, using the above counter-example, C.-J. Fu and B. Keller have discovered a counter-example (see $[\mathrm{FK}]$ ) to Conjecture 7.17 of [FZ3]. See also [C].

\section{REFERENCES}

[BFZ] A. Berenstein, S. Fomin and A. Zelevinsky, Cluster algebras III. Upper bounds and double Bruhat cells, Duke Math. J. 126 (2005), no. 1, 1-52.

[B] K. Bongartz, Tilted algebras, Representations of algebras (Puebla, 1980), Lecture Notes in Math., 903, Springer, Berlin-New York, 1981, (26-38).

[BCKMRT] A. B. Buan, P. Caldero, B. Keller, R. J. Marsh, I. Reiten and G. Todorov, Appendix to Clusters and seeds in acyclic cluster algebras, Proc. Amer. Math. Soc. 135, No. 10 (2007), 3049-3060. 
[BMR1] A. B. Buan, R. Marsh and I. Reiten, Cluster-tilted algebras, Trans. Amer. Math. Soc., 359, no. 1 (2007), 323-332.

[BMR2] A. B. Buan, R. J. Marsh and I. Reiten, Cluster mutation via quiver representations, preprint arxiv:math.RT/0412077v2, 2004, to appear in Comment. Math. Helv.

[BMRRT] A. B. Buan, R. J. Marsh, M. Reineke, I. Reiten and G. Todorov, Tilting theory and cluster combinatorics, Advances in Mathematics 204 (2) (2006), 572-618.

[BMRT] A. B. Buan, R. J. Marsh, I. Reiten and G. Todorov, Clusters and seeds in acyclic cluster algebras, Proc. Amer. Math. Soc. 135, No. 10 (2007), 3049-3060.

[CCS1] P. Caldero, F. Chapoton and R. Schiffler Quivers with relations arising from clusters $\left(A_{n}\right.$ case), Transactions of the American Mathematical Society 358 (2006), 1347-1364.

[CCS2] P. Caldero, F. Chapoton and R. Schiffler Quivers with relations and cluster-tilted algebras, Algebras and Representation Theory, 9, No. 4, (2006), 359-376.

[CK1] P. Caldero and B. Keller, From triangulated categories to cluster algebras, preprint math.RT/0506018 (2005), to appear in Invent. Math.

[CK2] P. Caldero and B. Keller, From triangulated categories to cluster algebras II, Ann. Sci. Ecole Norm. Sup, 4eme serie, 39, (2006), 983-1009.

[C] G. Cerulli Irelli, PhD-thesis in preparation: Structural theory of rank three cluster algebras of affine type.

[FZ1] S. Fomin and A. Zelevinsky, Cluster algebras I: Foundations, J. Amer. Math. Soc. 15 (2002), no. 2, 497-529.

[FZ2] S. Fomin and A. Zelevinsky, Cluster Algebras II: Finite type classification, Invent. Math. 154(1) (2003), 63-121.

[FZ3] S. Fomin and A. Zelevinsky, Cluster Algebras IV: Coefficients, Compositio Mathematica 143 (2007), 112-164.

[FK] C. Fu and B. Keller, On cluster algebras with coefficients and 2-Calabi-Yau categories, preprint arXiv:0710.3152 1 [math.RT], 2007.

$[\mathrm{H}]$ D. Happel, Triangulated categories in the representation theory of finite-dimensional algebras, London Mathematical Society Lecture Note Series, 119. Cambridge University Press, Cambridge (1988).

[HU] D. Happel and L. Unger, Almost complete tilting modules, Proc. Amer. Math. Soc. 107 (3) (1989), 603-610.

[K1] O. Kerner, Stable components of wild tilted algebras, J. Algebra 142 (1991), no. 1, 37-57.

[K2] O. Kerner, Representations of wild quivers, Representation theory of algebras and related topics (Mexico City, 1994), 65-107, CMS Conf. Proc., 19, Amer. Math. Soc., Providence, RI, (1996).

[MRZ] R. Marsh, M. Reineke and A. Zelevinsky, Generalized associahedra via quiver representations, Trans. Amer. Math. Soc. 355 (2003), no. 1, 4171-4186.

[R1] C. M. Ringel, Tame algebras and integral quadratic forms, Springer Lecture Notes in Mathematics 1099 (1984)

[Ri2] C. M. Ringel, The regular components of the Auslander-Reiten quiver of a tilted algebra, A Chinese summary appears in Chinese Ann. Math. Ser. A 9 (1988), no. 1, 102. Chinese Ann. Math. Ser. B 9 (1988), no. 1, 1-18.

[RT] I. Reiten and G. Todorov, unpublished.

[S] H. Strauss, On the perpendicular category of a partial tilting module J. Algebra 144 (1991), no. $1,43-66$.

INSTITUTT FOR MATEMATISKE FAG, NORGES TEKNISK-NATURVITENSKAPELIGE UNIVERSITET, N7491 TRONDHEIM, NORWAY

E-mail address: aslakb@math.ntnu.no

Department of Pure Mathematics, University of Leeds, Leeds, LS2 9JT, England

E-mail address: marsh@maths.leeds.ac.uk

INSTITUtT FOR MATEMATISKE FAG, NORGES TEKNISK-NATURVITENSKAPELIGE UNIVERSitet, N7491 TrondHEIM, NORWAY

E-mail address: idunr@math.ntnu.no 